\title{
Francesco Patrizi: Poetik und Psychologie des Staunens
}

\subsection{Biographische Notizen und Rezeptionsgeschichte}

Francesco Patrizi entwirft eine Poetik der maraviglia, die sich dediziert von den aristotelischen Prinzipien der Glaubwürdigkeit und der Nachahmung verabschiedet. Das Wunderbare (il mirabile) löst Patrizi von seiner kathartischen Funktion und konzipiert es als « forma $[. .$.$] e [. .$.$] anima della poesia ». { }^{1}$

Posto fine alle lunghe e noiose confutazioni da Aristotele alla poetica gittati, e delle non vere opinioni sopra ivi da gli spositori e da altri suoi seguaci fabricate, egli è tempo oggimai che noi altri insegnamenti più veraci dell'arte de' poeti e de' poemi a palesare incominciamo; per gli quali non solo la poetica arte si possa allo 'ntero far conoscere, ma possano i poeti ancora più sicuri a desiderato fin condurre i lor poemi. ${ }^{2}(D A$, II, 233)

Diese « insegnamenti più veraci dell'arte de' poeti e de' poemi » basieren auf der Definition des Staunens (maraviglia) und des Wunderbaren (mirabile) als Wesen der Poesie. Gewann Pontano seine Poetik der admiratio aus der Abgrenzung zu den Geschichtsschreibern und Rhetoren, entsteht Patrizis Poetik der maraviglia in der Auseinandersetzung mit aristotelischen und einer Vielzahl weiterer antiker wie auch zeitgenössischer Theorien. Zudem bindet Patrizi die eigene Erfahrung und logisch-rationale Überlegungen in

1 Danilo Aguzzi-Barbagli, Introduzione, in: Francesco Patrizi da Cherso, Della poetica, edizione critica a cura di Danilo Aguzzi-Barbagli, Bd. 1, Firenze 1969, S. XV.

2 Patrizi, Della Poetica, 3 Bde. Zur Poetica zählen die Deca istoriale (DI), die Deca disputata $(D D)$, die Deca ammirabile $(D A)$, die Deca plastica $(D P)$, die Deca dogmatica universale $(D D U)$, die Deca sacra $(D S)$ sowie die Deca semisacra (DSS). Zentral für unsere Untersuchung ist die Deca ammirabile. Die Zitate aus Della Poetica werden in der Folge im Lauftext mit der Angabe der Abkürzung der jeweiligen Deca sowie der Bandnummer und Seitenzahl aufgeführt. Die Übersetzungen aus Della Poetica stammen von der Autorin (A.E.): «Den langen und lästigen Widerlegungen des Aristoteles zur Poetik ein Ende bereitend, genauso den unwahren Meinungen, die seine Exponenten und andere seiner Anhänger hervorgebracht hatten, ist es jetzt an der Zeit, dass wir anderen beginnen, andere, wahrere Lehren über die Kunst der Dichter und der Dichtung zum Vorschein zu bringen; sodass nicht nur die poetische Kunst im Gesamten bekannt gemacht werden kann, sondern die Dichter ihre Werke noch sicherer dem gewünschten Ziel zuführen können. » 
seine Erkundung der Dichtung ein. ${ }^{3}$ Im Vordergrund stehen die verschiedenen Wirkungskategorien der Dichtung, wobei Patrizi sowohl das ästhetische, das Erkenntnis bringende als auch das religiöse Erlebnis betrachtet.

Della Poetica entstand während Patrizis Aufenthalt in Ferrara, wo er von 1578 bis 1592 einen von Alfonso II. D'Este errichteten Lehrstuhl für Platonische Philosophie innehatte. ${ }^{4}$ Patrizi zählte zum illustren intellektuellen Kreis der Estense und war dort mit diversen - auch delikaten - politischen und kulturellen Angelegenheiten betraut. ${ }^{5}$ Zuvor war Patrizi lange Jahre in Venedig als Sekretär im Dienste venezianischer Adliger tätig, er reiste mehrfach nach Zypern und perfektionierte dort seine Kenntnisse des Griechischen. Um 1570 weilte er unter Philipp II. in Spanien, bis er schliesslich als Professor nach Ferrara kam. 1592 wurde er vom Papst nach Rom berufen, wo er bis zu seinem Lebensende eine Professur für platonische Philosophie bekleidete. ${ }^{6}$

Patrizi war ein vehementer Kritiker des Aristotelismus. In seinen Discussiones Peripateticae vergleicht er aristotelische mit platonischen Standpunkten, zeigt auf, wo sie übereinstimmen und wo sie auseinandergehen, wobei er beinahe durchgehend die platonische Sichtweise favorisiert. ${ }^{7}$ Was er der Regelphilosophie des Aristoteles sowie den zeitgenössischen Bestrebungen nach einem Ideal der Ordnung und klassischen Kompositionsprinzipien entgegensetzt, wird er auch für seine Poetik einfordern: eine Rückkehr zur ursprünglichen

3 Patrizi gehörte zu den Wegbereitern der "neuen“ Wissenschaft sowie der Philosophie des 17. Jahrhunderts. Siehe Paul Oskar Kristeller, Eight Philosophers of the Italian Renaissance, London 1965, S. 112-113. Kristeller beschreibt einführend alle philosophischen Werke Patrizis. Vgl. dazu ebenfalls Carolin Hennig, Francesco Patrizis Della Poetica. Literaturtheorie der Renaissance zwischen Systempoetik und Metaphysik, Berlin 2016, S. 32-33.

4 Für eine ausführlichere Darstellung von Patrizis Werdegang siehe KRISTELler, Eight Philosophers, S. 113. Eine Auflistung aller Werke und Publikationen Patrizis findet sich in Anna Laura Puliafito, Francesco Patrizi da Cherso, in: Matteo Motolese et al., Il Cinquecento, Bd. 1, Salerno 2009, S. 297-307. Puliafito ist zudem die (Mit-)Herausgeberin folgender Werke Patrizis: Nova de universis philosophia: materiali per un'edizione emendata (1993) und Della retorica (1994). Ebenso hat sie mehrere Artikel zur Philosophie Patrizis, insbesondere zur Nova de universis philosophia, veröffentlicht.

5 Cesare Vasoli, Francesco Patrizi e il dibattito sul poema epico, in: Klaus W. Hempfer (Hg.), Ritterepik der Renaissance, Akten des deutsch-italienischen Kolloquiums Berlin 30.3.2.4.1987, Stuttgart 1989, S. 316, 319. Der Aufstieg Patrizis am Hof Ferraras fällt zusammen mit dem Abstieg Torquato Tassos.

6 ANNe BÉlAnger, Bomarzo ou les incertitudes de la lecture: Figure de la meraviglia dans un jardin maniériste du XVI ${ }^{e}$ siècle, Paris 2007, S. 233.

7 Kristeller, Eight Philosophers, S. 115. Diese detaillierte Gegenüberstellung von Platon und Aristoteles war eine wichtige und lange bewährte Grundlage späterer Aristoteles- und Platon-Forschung. 
Weisheit der Ältesten, ${ }^{8}$ und vor allem einen Fokus auf die «,magische Funktion des Sprechens »:9

[Patrizi sembra] affondare le sue radici in un pensiero che privilegia il potere magico ed evocativo della parola poetica. Nella Poetica egli attribuisce il linguaggio degli dei a quei poeti che attraverso la magia verbale suscitano uno stato di incantamento che trascende ogni distinzione della logica (vero o falso, reale o fantastico ecc.), per dispiegarsi liberamente nella ,meraviglia. La sua opposizione agli espositori cinquecenteschi della Poetica aristotelica si fonda, essenzialmente, su questa idea del poeta: ,facitore', cioè creatore di un cosmo letterario dove le parole sono le emblemi, ,geroglifici di una verità arcana alla quale può accedere soltanto il poeta divino che ha superato la distinzione fra logos e mithos attraverso il suo linguaggio mitpoietico. ${ }^{10}$

$\mathrm{Zu}$ poetologischen Fragen arbeitete Patrizi in verschiedenen Phasen seines Lebens: Im Jahr 1555 erschien sein Diskurs zu den unterschiedlichen Ausprägungen des poetischen Furors." Danach schrieb er selbst ein Poem (L'Eridano, 1557), ${ }^{12}$ kommentierte Ausgaben der Gedichte Luca Contiles (156o) und gab die Imprese von Girolamo Ruscelli (1572) heraus Er hielt eine Vorlesung zu Petrarca und 1583 - während der Niederschrift der Poetica - verfasste er eine Verteidigung des Orlando Furioso von Ariosto. ${ }^{13}$ Diese ist eine Antwort auf den Dialog Il Carrafa ovvero dell'epica poesia (1584) von Camillo Pellegrino, in der Pellegrino die poetischen Lehren des Aristoteles als objektiv-wissenschaftliche Prinzipien postuliert und die Gerusalemme Liberata Torquato Tassos aufgrund ihres elaborierten Stils, ihrer harmonisch-proportionalen Komposition sowie der Einheit ihrer Handlung als quasi perfektes Werk preist. Patrizi hingegen

8 Vasoli, Francesco Patrizi e il dibattito sul poema epico, S. 319. Vasoli zitiert aus Francisci PatriziI, Discussionum Peripateticarum tomi IV, quibus Aristotelicae philosophiae universa historia atque dogmata cum veterum placitis collata, eleganter et erudite declarantur, Basileae, ad Perneam Lecythum MDXXCI.

9 Michael Stausberg, Faszination Zarathustra. Zoroaster und die europäische Religionsgeschichte der frühen Neuzeit, Bd. 1, Berlin 1998, S. 298.

10 Walter Moretti, L'Ariosto di Francesco Patrizi, in: Patrizia Castelli (Hg.), Francesco Patrizi. Filosofo platonico nel crepuscolo del Rinascimento, Firenze 2002, S. 75.

11 Francesco Patrizi da Cherso, Discorso della diversità de i furori poetici allillustre Signor Mariano Savello, in: Patrizi, Della Poetica, Bd. 3, S. 447-462.

12 Vgl. dazu Lina Bolzoni, L’universo dei poemi possibili. Studi su Francesco Patrizi da Cherso, Roma 1980, S. 47; ebenso LuCiana Borsetto, Utopia, profezia, armonia. L'«Eridano» di Francesco Patrizi «in nuovo verso eroico», in: Studi Tassiani 45 (1997), S. 185 .

13 Diese schrieb Patrizi auf Anregung seines florentinischen Freundes Giovanni de' Bardi di Vernio. Patrizi war mit dem intellektuellen Umfeld von Florenz eng verbunden, unter anderem mit den «Accademie della Crusca e degli Alterati». Siehe VAsoli, Francesco Patrizi e il dibattito sul poema epico, S. 320. 
zeigt - in einem syllogistischen Verfahren - die Insuffizienz der aristotelischen Kriterien für eine Wissenschaft der Poesie sowie für die Literaturkritik auf.. ${ }^{14}$

Il Patrizi promise di scrivere sull'argomento un'opera che riaffermasse, in difesa di Ariosto, la libertà del poeta nel suo atto creativo, di contro ai vincoli e ai legami stabiliti dalla tradizione letteraria, in particolare, dai moderni espositori della poetica aristotelica. [...] Il Patrizi era convinto che i canoni della poetica aristotelica, proposti dal Pellegrino secondo il principio d'autorità, non potevano costituire, nella loro rigida e dogmatica formulazione, le fondamenta dell'attività poetica, creativa, perché non rispecchiavano l'uso né dei greci, né dei latini e, paradossalmente, non erano applicabili nemmeno ai poemi omerici, da cui, come da modelli, erano stati desunti. ${ }^{15}$

Seiner Poetik (Della Poetica) wandte sich Patrizi Ende der 1570er-Jahre zu und publizierte um 1586 die ersten beiden Deche. ${ }^{16}$ Wie sein Werk damals aufgenommen wurde, ist unklar. Während Danilo Aguzzi-Barbagli, Herausgeber von Della Poetica, davon ausgeht, dass Patrizis Position aufgrund seiner kontroversen Haltung gegenüber zeitgenössischen Kritikern und seiner radikalen Ablehnung des Aristotelismus relativ isoliert gewesen sei, ${ }^{17}$ bezeichnet Baxter Hathaway Patrizi als bereits zu Lebzeiten sehr einflussreichen und

\footnotetext{
14 VAsoli, Francesco Patrizi e il dibattito sul poema epico, S. 321.

15 Micaela Rinaldi, Il «Trimerone » di Francesco Patrizi fra epos e ,romanzo', in: Dies., Torquato Tasso e Francesco Patrizi. Tra polemiche letterarie e incontri intellettuali, Ravenna 20o1, S. 66. Patrizi nimmt in seiner Kritik von Pellegrinos Dialog keinen Bezug auf die Gerusalemme Liberata oder die Lyrik Tassos. Denn diese scheint Patrizi, entgegen der Poetik Tassos, nicht in das aristotelische Schema zu passen, sondern in ihrem Fokus auf die meraviglia, mit ihrer Verknüpfung von fantastischen mit realistischen Elementen, geschrieben in einem von der Alltagssprache entfernten, überraschenden und teilweise magisch-enigmatischen Stil, vielmehr seiner - Patrizis - Konzeption der Poesie nahe zu sein: « In questo suo testo il Patrizi non aveva mai fatto riferimento esplicito alla Liberata, alla quale, nonostante quanto era stato sostenuto dal suo autore in sede teorica, poco o nulla aveva a che fare - notava il Patrizi - con i canoni della poetica aristotelica e coni i presunti modelli omerici. [...] In realtà, egli [Patrizi] era profondamente consapevole della modernità dalla poesia tassiana e della sua altezza artistica („divino“ era per lui il Tasso come lo era l'Ariosto) [...] » Siehe Rinaldi, Il « Trimerone », S. 69-71.

16 Aguzzi-Barbagli, Introduzione, S. XII. Entgegen Patrizis Aussage, er habe die Reflexion zur Poetik in den Jahren von 1555 bis 1586 nicht nur unterbrochen, sondern vollständig aufgegeben (I, 8), kann davon ausgegangen werden, dass er sich in diesen Jahren intensiv mit den literaturkritischen und -theoretischen Texten seiner Zeit sowie mit klassischen Schriften zu Literatur und Rhetorik auseinandersetzte, so detailliert und umfangreich sind seine Kenntnisse.

17 Ebd., S. XIII. Auch Patrizis (natur-)philosophische Standpunkte, die eine originelle, aber ungewohnte Verbindung von literarischen und mathematischen Interessen aufweisen, hätten ihm zu seiner Zeit keine allzu grosse Anhängerschaft beschert, vermutet Aguzzi-Barbagli.
} 
erfolgreichen Denker. In einem aristotelischen Umfeld habe er die platonische Ästhetik weiterentwickelt, wobei er sein Augenmerk weg von der Mimesis, hin auf das Wunderbare und Staunenswerte der Dichtung gerichtet habe. ${ }^{18}$ Sein Einfluss habe aber kaum über seinen Tod hinaus angehalten, vielmehr sei das Interesse an ihm in einem hauptsächlich neoklassizistisch geprägten Umfeld verloren gegangen.

Heute sind insbesondere Patrizis philosophische Schriften aufgrund der « intrinsischen Qualität ihres Beitrags » und ihres Charakters als « Vorboten [...] frühneuzeitlicherWissenschaft und Philosophie » Inhalte der Forschung. ${ }^{19}$ Im Gegensatz zum philosophischen Hauptwerk, der Nova philosophia, wurde die Poetica bisher zwar wohlwollend erwähnt, aber nur marginal erforscht. ${ }^{20}$ Cesare Vasoli, der eine Monographie zu Patrizi verfasst hat, ${ }^{21}$ widmet der Poetica weder ein eigenes Kapitel noch einen eigenen Aufsatz - und dies, obwohl Vasoli sie als neuartigstes und originellstes poetologisches Werk des späten 15. Jahrhunderts einstuft. ${ }^{22}$ Nur Lina Bolzoni gibt eine umfassende Einführung in die philosophischen und poetologischen Schriften Patrizis, wobei sie insbesondere Della Poetica vertieft analysiert. ${ }^{23}$ Ihre Studie kann als Gegenpol zu Stefan Matuscheks Einschätzung von Patrizis Poetik der maraviglia gelesen werden: Matuschek spricht von einer Trivialisierung und Entleerung des Staunensbegriffs, von reiner Anhäufung verschiedener Ideen und Zitate ohne schlüssiges Konzept, vom far stupire aus purer Effekthascherei, ${ }^{24}$ während Bolzoni betont, dass bei Patrizi die Begriffe des mirabile und der maraviglia

18 BaXter Hathaway, Marvels and Commonplaces, New York 1968, S. 65.

19 Kristel Lle R, Eight Philosophers, S. 112.

20 Vgl. Weinberg, A History; Chevrolet, Lidée de la fable; Hathaway, Marvels; Castelli, Francesco Patrizi.

21 Cesare Vasoli, Francesco Patrizi da Cherso, Roma 1989; ebenso Ders., Schede patriziane sul De Sublime, in: Giovanni Casertano, Antonio Capizzi und Giuseppe Martano (Hgg.), Il sublime. Contributi per la storia di un'idea. Studi in onore di Giuseppe Martano, Napoli 1983, S. 161-173; und De Rs., Francesco Patrizi e il dibattito sul poema epico, S. 315-332.

22 VAsoli, Francesco Patrizi e il dibattito sul poema epico, S. 316. Auch Kristeller mass der Poetica hohen Wert bei, ohne sie eingehend zu untersuchen. Siehe Kristeller, Eight Philosophers, S. 114.

23 Im deutschen Sprachraum wurden fast ausschliesslich die philosophischen Schriften Patrizis rezipiert, zum einen seine detaillierte Gegenüberstellung platonischer und aristotelischer Prinzipien in den Discussiones peripateticae (1581), zum anderen gilt er als «Figur ersten Ranges [...] für eine Zoroaster-Rezeptionsgeschichte». Laut Michael Stausberg hat Patrizi « den von Ficino inaugurierten Zoroaster-Diskurs zu seiner letzten Konsequenz geführt », bevor zu Beginn des 16. Jahrhunderts neue Deutungsversuche einsetzten. Siehe Stausb R R, Faszination Zarathustra, S. 291-292. 
ausgearbeitet, ihre Genese wie auch ihre Bedeutungsspektren weitreichend aufgezeigt würden. ${ }^{25}$ Matuscheks Einschätzung wird auch deshalb zu modifizieren sein, weil Patrizi in der Poetica seine Vorstellung der Dichtung als Rückkehr zu kultischem Gehalt klar umreisst. Diese Dichtung lässt göttliche Weisheit erahnen und transportiert (Ur-)Wissen und zivilisatorische Werte.

Anne Bélanger untersucht Patrizis Poetica im Rahmen ihrer Studie über Vicino Orsinis Sacro Bosco in Bomarzo und führt eine Nähe beider Werke zur Hypnerotomachia Poliphili wie auch zum Orlando Furioso ins Feld. Das Vorgehen ist insofern anachronistisch, als der Garten in Bomarzo vor der Niederschrift der Poetica entstand - der Fokus liegt denn auch nicht auf der historisch belegbaren, sondern auf der inhaltlichen Nähe der genannten Werke. Diese Nähe liegt in der Originalität der Werke begründet, in ihrem Fokus auf die Rezipienten bzw. deren Staunen über das Kunstwerk als Anregung der Imagination, in der Betonung des Schöpferischen und Fiktiven, in der Hervorhebung des magischen, wundersamen Gehalts der Sprache bzw. des Kunstwerkes sowie in der bewussten Abwendung vom Postulat der Nachahmung. ${ }^{26}$

Von Bolzoni und Bélanger ausgehend versuche ich in diesem Kapitel aufzuzeigen, welchen Stellenwert die Begriffe mirabile und maraviglia in der Poetica Patrizis einnehmen, wie sie definiert und wie sie zur Bestimmung der Dichtung und des Dichters herangezogen werden. Zudem stellt sich die Frage, inwiefern Patrizi als Kulminationspunkt einer Poetik des Staunens betrachtet werden kann und wie er sich zu seinen Vorgängern Pontano, Fracastoro und Vida, die er mitunter in der Poetica zitiert, stellt.

\subsection{Patrizi zwischen Aristoteles und Platon}

Mit seiner Konzeption der Dichtung bricht Patrizi mit dem aristotelischen Zirkel, deren neoklassizistisches Interesse vor allem auf die Tragödie und das Epos gerichtet war. ${ }^{27}$ Patrizi moniert, diese literarischen Genres seien erst viele Jahre nach dem Ursprung der Poesie entstanden. Zu den ursprünglichen Genres hingegen zählen - inspiriert vom göttlichen Furor -: Orakel, Prophezeiungen, Hymnen, Gebete, Rätsel und Orgien. ${ }^{28}$ Für diese Genres sind

25 Bolzoni, L'universo, S. 123.

26 BÉlANger, Bomarzo, S. 233-248. Zum selben Thema ebenfalls empfehlenswert Raffaella Fabiani Giannetto, „Not before either known or dreamt of": The Hypernotomachia Poliphilii and the craft of wonder, in: Word \& Image 31 (2015), S. 112-118.

27 HathaWAY, Marvels, S. 68.

28 Renato Barilli, Poetica e retorica, Milano 1969, S. 133. 
die Prinzipien der Mimesis ${ }^{29}$ und der Handlung bedeutungslos, weshalb Patrizi sie nicht als grundlegende Elemente der Poetik ansieht. Zur epica poesia zählt Patrizi demnach nicht nur das poema eroico, sondern das Sprechen in Versen allgemein. ${ }^{30}$ Neben der historisch belegten literarischen Vielfalt ist es die Unerschöpflichkeit der menschlichen Natur mitsamt den unterschiedlichen Impulsen und Widersprüchen, die ein einheitliches Regelsystem unmöglich macht. $^{31}$

Fast stärker noch als Aristoteles selbst kritisiert Patrizi die Aristoteliker seiner Zeit - vor allem Iacopo Mazzoni und Ludovico Castelvetro -, und zwar nicht hauptsächlich aufgrund ihrer aristotelischen Basis, sondern weil sie ihre Autoritätsgläubigkeit über ihre eigenen Erfahrungen und Überlegungen stellen würden. ${ }^{32}$ Ein Dorn im Auge ist ihm auch die Verknüpfung des mirabile mit dem Glaubwürdigen, Möglichen, Wahrscheinlichen. ${ }^{33}$ Patrizi wirft Mazzoni und Castelvetro vor, eine ganze Anzahl antiker Quellen, die das genaue Gegenteil postulieren, ausser Acht zu lassen:

Che sia l'incredibile il vero fondamento del meraviglioso è ampiamente attestato presso gli antichi: Patrizi ricorre all'autorità di Dion Crisostomo, di Palefato, di Ermogene. Le fonti del ,mirabile' risultano, ad una più ampia disamina degli autori antichi, tutte legate all'incredibile. ${ }^{34}$

Dennoch muss Patrizis Auseinandersetzung mit den Aristotelikern differenziert betrachtet werden. Aristoteles ist nur ein - wenn auch wichtiger Bezugspunkt unter vielen: Patrizi setzt sich auch mit den Schriften Platons, dem Corpus Hermeticum und weiteren griechischen Autoren wie Strabon, Dion Chrysostomos und Plutarch auseinander - beispielsweise um seine Überlegungen zu zeremoniellen lyrischen Genres oder der poetischen Inspiration zu untermauern.

29 Angela Andrisano bemerkt richtigerweise, dass Patrizi sich ein relativ eindimensionales Bild der mimesis machte und er diese nur als Prinzip der Nachahmung und der Ähnlichkeit verstand. Siehe ANg ELA ANdrisano, Patrizi e il,meraviglioso. Le fonti classiche, in: Castelli, Francesco Patrizi, S. 67. Vgl. dazu auch DD, II, S. 78.

30 Francesco Patrizi da Cherso, Parere di Francesco Patrizi in difesa di Lodovico Ariosto, in: Gianni Gelati (Hg.), Ludovico Ariosto, Roma 2009, S. 1281-1282. Vgl. ebenso DD, II, S. 94.

31 Moretti, L'Ariosto, S. 75 .

32 AguzZi-BArbagli, Introduzione, S. IX.

33 Peter G. Platt, ,Not before either dreamt or known of'. Francesco Patrizi and the Power of Wonder in Renaissance Poetics, in: The Review of English Studies 43 (1992), S. 39 o.

34 Andrisano, Patrizi, S. 7o. 
Da Patrizi sich ausführlich mit den Schriften Platons befasste und dessen Philosophie lehrte - auch, weil der furor poeticus und das Göttliche in Patrizis Poetik eine grosse Rolle spielen -, wird er vielfach als Platoniker bezeichnet. ${ }^{35}$ Bei genauerem Hinsehen wird man jedoch gewahr, dass Patrizi weder vollumfänglich als Anhänger Platons noch als durchgängiger Kritiker Aristoteles' bezeichnet werden kann. ${ }^{36}$ Oftmals werden Aussagen Platons modifiziert oder nur teilweise übernommen. So ist es als grundlegende Modifikation zu begreifen, dass Patrizi die Kunst nicht wie Platon und Ficino als Abbild der Idee und der Realität und damit als unvollkommen begreift, sondern - im Gegenteil - als ursprünglichen Ausdruck der (göttlichen) Weisheit versteht. ${ }^{37}$ Im Eridano, der am Ende dieses Kapitels analysiert wird, greift Patrizi auf Proteus als prophetische Figur zurück - während Platon in der Politeia wie auch im Ion den Mythos von dessen Verwandlungen als Lügengeschichte zurückweist. ${ }^{38}$ Genauso gibt es einzelne Elemente, die Patrizi aus Aristoteles' Philosophie übernimmt, obwohl er zumeist bemüht ist, dessen Positionen als unzulänglich darzustellen. Beispielsweise ist Patrizis Konzeption der Seele (anima) viel stärker an Aristoteles denn an Platon orientiert. ${ }^{39}$

35 Patrizi bezieht sich insbesondere auf folgende Werke Platons: Politeia, Nomoi, Sophistes, Phaidros, Ion und Symposion. Siehe AguzzI-Barbagli, Introduzione, S. VII. Eine ausführliche philosophische Untersuchung zu Patrizis Neuplatonismus mit vielen Quellenverweisen findet sich in Thомas Leinkauf, Il neoplatonismo di Francesco Patrizi come presupposto della sua critica ad Aristotele, Firenze 199o. Leinkauf thematisiert insbesondere die Verbindung von «platonismo cristiano » und «filosofia speculativa della natura » (S. 72), die in dieser Form einzigartig ist.

36 Vgl. dazu Hennig, Francesco Patrizi, S. 34.

37 Aguzzi-Barbagli, Introduzione, S. VIII: « [...] in nome dell'esperienza e della ragione il Patrizi non esiterà a respingere il bene amato Platone. [...] Sempre sotto il medesimo impulso sperimentativo e raziocinante il Patrizi non esita talvolta ad incorporare principi aristotelici, ad attingere a larga mano dal Sublime dello pseudo-Longino [...], da Plutarco, da Ermogene e dai retori greci, dai geografi e dagli storici, da Aristide, Qunitiliano e dalla tradizione musicografica, non esclusi persino gli rapporti bizantini. » Vgl. auch Andrisano, Patrizi, S. 65, 71; ebenso Fabrizio Desideri und Chiara Cantelli, Storia dell'estetica occidentale. Da Omero alle neuroscienze, Roma 2008, S. 169.

38 Francesco Patrizio, L'Eridano. Allo illustrissimo e reverendissio cardinale di Ferrara, in: Giosué CARducci, La poesia barbara nei secoli XV e XVI, ristampa anastatica 1985 dell'edizione 1881 con presentazione di Emilio Pasquini, Bologna 1985, S. 330, V. 124. Vgl. dazu Polit., 381d, und Ion, 541e.

39 Vgl. Kapitel 6.4.4. Für Platon ist die Seele das unsterbliche Prinzip, das vom Körper unabhängig und diesem übergeordnet ist. Für Aristoteles ist die Seele die Vollendung des Körpers, der als Materie das Lebendigsein zwar potenziell in sich trägt, dieses aber erst durch die Seele entfaltet wird. 
Patrizis Umgang mit antiken und zeitgenössischen Texten kann als eklektisches Beiziehen unterschiedlicher Elemente in die eigenständige Reflexion beschrieben werden:

[...] l'elemento che distingue il Patrizi tra la folla dei critici cinquecenteschi è questo spirito libertario $[\ldots]^{40}$

\subsection{Der Aufbau von Della Poetica}

Francesco Patrizi wagt in seiner Poetik eine neue Form der Literaturbetrachtung. Allerdings bildet sie nicht, wie Renato Barilli und Danilo AguzziBarbagli postulieren, einen irrationalen und gefühlsbetonen Gegenpol in einem zunehmend rationalistisch geprägten Umfeld, sondern eine durchaus rational und logisch begründete wie auch auf Erkenntnis zielende Formulierung universell gültiger Prinzipien einer Wissenschaft der Dichtung. ${ }^{41}$

Patrizi leitet seine Überlegungen von einem historisch-mythischen Bezugspunkt ab: dem antiken, vorhomerischen Griechenland als Ursprungsland der Poesie und der Kultur. Aus dieser Zeit sind nur wenige, meist fragmentarische Schriften überliefert - eine fruchtbare Ausgangslage für die Kreation eines Ursprungsmythos, der zu gleichen Teilen auf Überlieferung wie auf Fiktion beruht. ${ }^{42}$

One result of this insistence, throughout the volumes, upon the earliest examples is the impression that this is a poetics based upon the prehistory of poetry, a poetics founded upon Orpheus. [...] For the poems which Patrizi studies most abundantly seem to be poems which do not exist, which we know only through the reports of other ancient authors. Patrizi is thus free to conjecture what their content might have been, should have been, must have been. The theoretical presuppositions thus come to be read into the historical facts. ${ }^{43}$

Lina Bolzoni deutet Patrizis Vorgehen als «Überspringen der historischen Dimension», als «Sich-Einfinden auf einer metaphysischen Ebene ». ${ }^{44}$ Viel wichtiger als der metaphysische scheint mir allerdings der mythische

\footnotetext{
40 AguzZI-BARbagli, Introduzione, S. VIII.

41 BARILli, Poetica eretorica, S. 128; A gUZZI-BARbagli, Introduzione, S. XIV-XV; BolzonI, L'universo, S. 7 .

42 Eine nur untergeordnete Rolle spielen die literarischen Texte des Mittelalters und des Humanismus; sie werden zwar kurz erwähnt, aber in wenigen Seiten abgehandelt. Siehe Bolzoni, L'universo, S. 116.

43 Weinberg, A History, S. 782.

44 Bolzoni, L'universo, S. 67.
} 
Charakter zu sein, der dem Ursprung der Dichtung zukommt. Er ist kein historischer Fakt, sondern von Anfang an ein literarisches Artefakt. So schöpft sich Patrizi seinen Mythos der Entstehung, der Geschichte sowie des Wesens der Poesie in Analogie zu den antiken Theogonien und Kosmogonien wie der Orphischen Argonautika, die zudem wichtige Bezugstexte von Della Poetica darstellen. Dem Bezug auf früheste literarische Zeugnisse liegt auch die Überzeugung zugrunde, dass deren Sprache magische Kräfte besass, sie nicht die Welt abbildete, sondern den Gang der Welt und des Kosmos steuerte. ${ }^{45}$

Die Poetica Patrizis besteht aus sieben Deken. ${ }^{46}$ In den ersten beiden Deche, der Deca istoriale (DI) und der Deca disputata $(D D)$, zeichnet Patrizi zum einen die Frühgeschichte der Poesie nach und klassifiziert die auftretenden Genres, zum anderen setzt er sich kritisch mit den Positionen der Aristoteliker auseinander - vor allem mit deren Positionen in Bezug auf Mimesis, Handlung und Vers. ${ }^{47}$ Patrizi verwirft die gängigen aristotelisch geprägten Ansichten, dass Poesie Nachahmung und der Poet ein Nachahmer sei, dass die Handlung das eigentlich poetische Handwerk sei, dass der Stoff entsprechend nur eine « in sich geschlossene Handlung mit Anfang, Mitte und Ende $»^{48}$ beinhalten und nicht aus der Geschichte oder Wissenschaft stammen dürfe ${ }^{49}$ und dass Rhythmus und Harmonie keine eigenständigen Elemente, sondern lediglich Mittel zum Zweck der Nachahmung seien.

Falsi sono adunque, e da verità molto lontani, questi aristotelici insegnamenti che la poesia imitazione sia e che la favola sia imitazione nè d'azione, nè d'altra cosa; e falso è parimenti che perciò il poeta sia favoleggiatore, più tosto che verseggiatore, nè quanto per imitazione egli è poeta $[\ldots]^{50}(D A, \mathrm{II}, 282)$

45 Vasoli, Francesco Patrizi da Cherso, S. 94.

46 „Deka“ steht für griechisch „zehn“: Jede Deka besteht aus zehn Büchern.

47 Weinberg, $A$ History, S. 767: «The Deca disputata, in fact, is a "negative poetics“, which centers primarily about these two matters, imitation and verse.»

48 Poet., 1459a.

49 Aristoteles klammert damit Stoffe aus der Geschichtsschreibung, der Kunst und der Wissenschaft aus den möglichen literarischen Stoffen aus. Denn in der Geschichtsschreibung werden ganze «Zeitabschnitt[e]» mit mehreren Handlungen dargestellt. Sachthemen der Kunst und der Wissenschaft kommen ganz ohne Handlung aus und sind dadurch ebenso vom aristotelischen Stoffrepertoire ausgeschlossen. Weinberg umschreibt die Problematik der aristotelischen Einschränkung auf die Handlung folgendermassen: «[...] by limiting poetry to imitation one limits it to plot and [...] this in turn confines its scope to human actions, passions, and character. But essentially the field of poetry ist much broader than this; it admits no restrictions; it is coincident with the fields of history and of the natural sciences and of all forms of knowledge. » Siehe Weinberg, A History, S. 769 .

50 Falsch sind also, und sehr weit von der Wahrheit entfernt, diese aristotelischen Lehren, dass die Dichtung Nachahmung sei und dass die Erzählung Nachahmung der Handlung 
Diese negative Poetik der Deca disputata, zusammen mit einer Darstellung der vorhomerischen griechischen Literatur in der Deca istoriale, bildet die Ausgangslage der späteren Deche.

Diese fünf späteren Deche ${ }^{51}$ lagen bis zur Ausgabe von 1969 nur handschriftlich vor und weisen einen teilweise fragmentarischen Charakter auf. Paul Oskar Kristeller entdeckte die Manuskripte 1949 dank eines Hinweises von Ervino Zorzi in der Biblioteca Palatina di Parma und stellte sie als Mikrofilm für die Edition von 1969 zur Verfügung. Kristeller geht davon aus, dass die Manuskripte den Zeitgenossen von Patrizi nicht bekannt waren, Bélanger hingegen nimmt an, dass sie in gelehrten Zirkeln kursierten. ${ }^{52}$ Einig sind sich Kritiker und Forscher darin, dass sie den eigenständigen und originellen Teil von Patrizis Poetik überliefern. ${ }^{53}$

Die Deca ammirabile $(D A)$ ist die erste der fünf unpublizierten Deken. Sie ist der systematische Versuch, eine Poetik der maraviglia und des mirabile zu konzipieren. ${ }^{54}$ Die Koppelung von mirabile und maraviglia - die wunderbare Beschaffenheit der Dichtung, die Staunen auslöst - bestimmt das Wesen der Dichtung vom Ursprung bis hin zu deren Rezeption.

In den folgenden Deken werden die spezifischen Einzelgattungen sowie die Definitionen von Form und Materie abgeleitet. ${ }^{55}$ Die Deca plastica (DP) handelt, wie der Name bereits andeutet, von der Formung und Herstellung des mirabile, als dessen Grundelemente Transformation und Fiktion - und damit einhergehend das Neue - gelten: ${ }^{56}$

[la finzione] altro non è che dare ad una cosa forma diversa da quella che havea prima ed apparenza: ciò è una forma nuova, o rinnovata. ${ }^{57}(D P$, III, 19)

oder einer anderen Sache sei; ebenso ist es falsch, dass der Dichter viel eher Erzähler als ein Komponist von Versen sei, und dass er Dichter sei aufgrund der Nachahmung.

51 La Deca ammirabile, La Deca plastica, La Deca dogmatica universale, La Deca sacra, La Deca semisacra.

$5^{2}$ BÉlANGER, Bomarzo, S. 235.

53 AguZZI-BARbaG LI, Introduzione, S. XIII-XIV.

54 Ebd., S. XV.

55 Weinberg, A History, S. 775. Für eine ausführliche Beschreibung von Patrizis Gattungssystematik siehe Hennig, Francesco Patrizi, S. 101-134.

56 Patrizi siedelt die Transformation auf allen Ebenen der Dichtung an: angefangen beim Wortmaterial, weiter zu den Tropen - wobei insbesondere « die Metapher und figuratives Sprechen [...] im Zentrum» stehen -, hin zur Versbildung und zum Inhalt. Siehe dazu Hennig, Francesco Patrizi, S. 177.

57 Die Fiktion ist nichts anderes, als einer Sache eine Form zu geben, die sich von ihrem früheren Erscheinungsbild unterscheidet: Es ist eine neue oder erneuerte Form. 
Ed in somma tutta la poesia, secondo i gradi delle materie sue, più o meno dee con finzone essere detta, e senza finzione non mai, poscia che doppio e triplo è il mirabile che accompagna e la maraviglia che la segue. E qual poema meno hebbe di finzione, meno anco ebbe di maraviglia e meno consegue il suo fine. ${ }^{58}$ (DP, III, 26)

Im Unterschied zu anderen Formen der Sprache wird in der Dichtung das Material so geformt und modelliert (plasmato), dass es Staunen erzeugt. ${ }^{59} \mathrm{Am}$ transformationalen Charakter der Dichtung bemisst sich neben dem Grad des Staunenswerten auch die Kreativität des Dichters. ${ }^{60}$

In der Deca dogmatica universale $(D U)$ werden nochmals allgemeine Bereiche der Poetik besprochen - vom Titel des Poems über die Invokation der Musen zum (Er-)Finden des Stoffes -, bevor mit der Deca sacra (DS) eine detaillierte Diskussion der Genres und Subgenres einsetzt. Diese ist orientiert an den Stoffen, die entweder göttlich, natürlich oder menschlich (oder gemischt) sind, sowie an der Geschichte der einzelnen Genres. ${ }^{61}$ Damit geht eine Wertung der einzelnen Genres einher, wobei die ältesten und die religiösesten die ehrwürdigsten sind. ${ }^{62}$ Wie in der Deca istoriale werden grösstenteils Genres besprochen, die nicht mehr zu den gängigen Formen der Dichtung zählen.

\subsection{Die Deca ammirabile}

Für die Deca ammirabile, die für uns von besonderer Relevanz ist und auf die ich im Folgenden vertieft eingehe, stehen literaturtheoretische Überlegungen im Vordergrund. Die Deca ammirabile ist in zehn Bücher gegliedert und führt von den akzidentiellen zu den notwendigen, wunderbaren Eigenschaften der Poesie («proprietà poetiche [...] mirabili »), weiter zum Poeten als wunderbaren Schöpfer («mirabile facitore») und schliesslich - als Klimax - zu den Definitionen des «mirabile» und der «maraviglia» als wesentliche Eigenschaften und Bestandteile der Dichtung.

$5^{8}$ Jede Dichtung also muss, entsprechend ihren Stoffen, mehr oder weniger fiktiv sein und darf nie ohne Fiktion auskommen, denn doppelt oder dreifach sind das Wunderbare, das sie begleitet, und das Erstaunliche, das ihr folgt. Und ein Poem, das weniger Fiktion enthält, enthält auch weniger Erstaunliches und folgt seinem Ziel entsprechend weniger.

Bolzoni, Luniverso, S. 128.

6o Ebd., S. 131.

61 WeINBERG, A History, S. 782.

62 Ebd., S. 782. 
Patrizi lehnt es ab, die Poetik als eine Spezialform der Rhetorik zu betrachten, und sucht wie schon Pontano und Fracastoro gezielt nach den „eigentlichen“ und „eigentümlichen“ Eigenschaften der Poesie (DA, II, 233) ${ }^{63}$ Allerdings richtet sich Patrizis Fokus nicht auf eine Eigenschaft, die nur der Dichtung zukäme, sondern auf poetische Qualitäten, die ausnahmslos allen poetischen Erzeugnissen - allen Gattungen und Stilrichtungen, allen historischen wie auch allen zukünftigen Dichtungen - zukommen.

Anhand der Datierung zeigt sich, dass die zehn Bücher der Deca ammirabile in einem sehr kurzen Zeitraum (April-Juni 1587) entstanden. Spannend ist, dass das letzte Buch mit dem Titel «Che cosa sia la maraviglia» («Was das Erstaunliche sei») - die Essenz der gesamten Deca - als Erstes entstand (23. April 1587) und die herleitenden Kapitel erst danach verfasst wurden. Patrizi inszeniert die Gewinnung seiner Thesen und Begriffe als Herleitung, letztlich dienen seine Reflexionen und die historischen Beispiele aber vor allem der Bekräftigung seiner Grundthesen, die von Anfang an feststehen.

[...] l'analisi della "natura“ della poesia prevalga sulla deduzione dalla storia, alla quale fornisce materiale di exempla, strumenti di conferma, piuttosto che i fondamenti dell'elaborazione teorica. ${ }^{64}$

In diesem Sinne ist denn auch Weinbergs Schilderung von Patrizis Vorgehen zu lesen:

We may summarize his procedure, briefly, thus: At any point where a distinction is needed, whether as a first principle or as a derivation or even as a defnition, he begins by collecting a large number of statements relevant to the distinction, made by an ancient (and occasionally by modern) theorists. He then summarizes all these statements $[\ldots]$, groups and categorizes them, finally reduces them to a single resumptive one, which is the answer to his present problem. This process is frequently followed by an examination of conflicting theses, refuted one by one by an appeal to additional passages and to the example of the poets. We should note, however, that the collection of statements is never in itself completely random. ${ }^{65}$

63 Patrizi lehnt das Wiederaufleben der antiken Rhetorik durch die Humanisten als Gesamtes ab, da es «un frutto fuori stagione» sei. Sie sei angebracht gewesen in den antiken Republiken, gehöre aber nicht in die Zeit der Renaissance mit ihren Monarchien und Herrschaften. Der rhetorischen Sprache geht es um Überzeugung und Macht, nicht um Wissen. Demgegenüber schlägt Patrizi eine Reform der Rhetorik vor: vom politischen Machtinstrument zu einer « Doktrin des , menschlichen Redens » , dessen Fundament die Natur, das Wissen, das Wahre wären. Siehe Vasoli, Francesco Patrizi da Cherso, S. 105, 107.

64 Bolzoni, L'universo, S. 12 o.

65 Weinberg, A History, S. $77^{2}$. 
Als « statements » aus anderen Texten werden nie ganze Reflexionsbögen eingebunden, sondern immer nur kurze Zitate - manchmal nicht einmal vollständige Sätze, die zudem meist aus ihrem Kontext enthoben eingebunden und kombiniert werden. Sie stehen im Dienst von Patrizis Überlegungen nicht umgekehrt.

Auf der Suche nach den spezifisch dichterischen Qualitäten akkumuliert Patrizi im ersten Buch vierzig Eigenschaften, zusammengetragen von verschiedenen Autoren und Kontexten. Diese reduziert er auf sieben Eigenschaften, die nur der Poesie und keiner anderen Textform zukommen: «Enigma, Vielfalt, Grösse, Sanftheit, verfremdete Sprache, Vers, Gesang » ( « enimma, varietà, grandezza, dolcezza, lingua straniera, verso, canto », $D A, \mathrm{II}$, 255) ${ }^{66}$ Noch immer aber ist die Frage ungeklärt, welches denn jene essenzielle Eigenschaft ist, die ausnahmslos allen poetischen Zeugnissen inhärent ist:

The answer is that such a quality does exist and that it is the "mirabile“, that at which we wonder and marvel. Again the authorities are consulted, and we learn that each of the seven common properties of poetry is by itself marvelous. ${ }^{67}$

Auch das Pendant des mirabile - die maraviglia - wird anhand verschiedener Autoren, Kontexte und Kategorien bestimmt. Für Platon und Aristoteles kann Nichtwissen eine Quelle des Staunens sein, für Aristoteles, Plutarch und Strabon sind es zudem überraschende Momente einer Handlung. Dieselben Autoren bezeichnen auch das Neue als staunenerregend, für PseudoLongin resultiert das Staunenswerte aus dem paradoxon. ${ }^{68}$ Patrizi führt diese Positionen zusammen und begründet die Quelle des Staunenswerten schlussendlich nicht nur im Neuen, Unglaublichen oder Überraschenden, sondern auch im Zusammenspiel dieser Qualitäten mit ihren Gegensätzen: mit dem Wahrscheinlichen, Glaubwürdigen und Erwartbaren.

\subsubsection{Der Dichter als facitore del mirabile und mirabile facitore}

Mirabile und maraviglia müssen an ihre Entstehung und insbesondere an den vom göttlichen Furor durchströmten Dichter rückgebunden werden: Der Dichter ist «Schöpfer des Wunderbaren » («facitore del mirabile») und « wunderbarer Schöpfer» («mirabile facitore ») gleichermassen. ${ }^{69}$

66 Weinberg, A History, S. 772.

67 Ebd., S. 773 .

68 Andrisano, Patrizi, S. 70. Das paradoxon schliesst nicht nur das Widersprüchliche, sondern allgemeiner das Unerwartete, Ausserordentliche und Unglaubliche ein.

69 Weinberg, A History, S. 773. 
Insofern als er Wunderbares schafft, kreiert er Neues, nie Dagewesenes. Angelehnt an die griechische Bedeutung von poésis («Erschaffung») wird der Dichter als Schöpfer («facitore») konzipiert, und zwar in erster Linie als Schöpfer von Versen («poeta è il fattore di versi », $D A$, II, 274), nicht als Schöpfer von Nachahmungen der menschlichen Handlung («imitatore») oder der Idee (« effigiatore») im platonischen Sinne $\left(D A\right.$, II, 275) $\cdot{ }^{70}$ Entsprechend zeichnet sich der Poet nicht durch die Auswahl des Stoffes aus, sondern durch die poetische Bearbeitung von diesem:

Il che non tanto sta nella materia, o nel trovarla o non trovarla, quanto nel trattarla con poetiche proprie maniere $[\ldots]^{71}(D D$, II, 162$)$

Um Wunderbares zu schaffen, muss der Poet vom mirabile erfüllt sein. Dieses trägt er zum einen in sich selbst, zum anderen wird es ihm von Gott eingeflösst. Eine entsprechende Theorie dazu hat Patrizi in der Deca disputata entwickelt, und zwar anhand der Enthusiasmus-Theorien ${ }^{72}$ von Aristoteles und Platon. Aristoteles definiert den Enthusiasmus als Affekt und stellt ihn auf die gleiche Ebene wie die Angst und das Mitgefühl. Diese Definition ist für Patrizi unzureichend, denn der poetische Enthusiasmus kommt nur wenigen zu, während die Angst und das Mitgefühl allen Menschen gemein ist $(D D$, II, 24).

Für Platon ist der Furor nicht in der Natur des Menschen angelegt, sondern wird ihm von göttlicher Instanz oder von den Musen eingegeben. In diesem Sinne ist der Poet aber lediglich ein Sprachrohr für einen Text, den er ohne eigenes Zutun von sich gibt. Auch diese Sicht ist für Patrizi nicht zufriedenstellend, da er den Poeten als eigenmächtigen Schöpfer von Dichtung

70 Laut Ossola ist das späte 16. Jahrhundert durch den Streit um die richtige Art der Nachahmung - der Natur oder der Idee - geprägt. Es wäre sicherlich lohnenswert, Patrizi in diese Kategorisierung miteinzubeziehen, da er einen Gegenpol zur Nachahmung in allen ihren Ausprägungen markiert und in dieser Rolle den Blick auf die Geschichte der Literaturtheorie differenzieren würde. Siehe CARLo Ossola, Autunno del Rinascimento. «Idea dell'tempio » dell'arte nell'ultimo Cinquecento, Florenz 1971. Der Hinweis auf Ossola stammt von Gerhard Regn, Tasso und der Manierismus. Anmerkungen zu einem Forschungsproblem, in: Romanistisches Jahrbuch 38 (1987), S. 111.

71 Es geht nicht so sehr um den Stoff, oder darum, diesen zu finden oder nicht, sondern ihn mit poetischen Verfahren zu behandeln [...]

72 „Enthusiasmus“ und „Furor“ werden von Patrizi als Synonyme verwendet; Ersterer ist aber mehr im Menschen angelegt, während Letzterer eher von Gott zu kommen scheint. 
anerkennt und in den Dichtern den Ursprung des Wissens und der Weisheit angelegt sieht. ${ }^{73}$

Die Verbindung beider Positionen findet er bei Strabon und Plutarch und schliesst sich dieser an:

Così quello che è chiamato entusiasmo pare essere una mescolanza di due movimenti: l'uno per lo quale l'anima pate, l'altro per lo quale ella si muove secondo natura. ${ }^{74}(D D, \text { II, } 27)^{75}$

$\mathrm{Zu}$ diesem Schluss ist Patrizi bereits in seiner frühen Schrift Discorso della diversità de i furori poetici $(D F)$ gelangt: «ci desidera due condizioni, l'una dell'ingegno e l'altra del furore, a fare perfetto il poeta $»^{76}(D F$, III, 449). Der «ingegno » stellt eine bestimmte Haltung des menschlichen Geistes dar, jene des Lernens und Wiederfindens («attitudine [...] all'imparare e al ritrovare», $D F$, III, 449), während der poetische Furor - entsprechend Platons Konzeption im Phaidros und im Ion - ein göttlicher ist. Menschliches und göttliches Wahrnehmen, Erkennen und Darstellen werden eins im Dichter. ${ }^{77}$

Menschliches und Göttliches vereinen sich auch in der Konzeption des Schöpfers, der an die drei universellen Schöpfergestalten Platons angelehnt ist: Gott, die Natur sowie den Künstler (DA, II, 285). Der Dichter als Schöpfer so Patrizi - ist nicht reduziert auf die Rolle des Letzteren, sondern hat - in seiner höchsten Ausprägung - darüber hinaus Anteil am Göttlichen sowie an der Natur und wird zur verbindenden Figur der drei Bereiche. ${ }^{78}$

73 Richtig und wichtig scheint mir Lina Bolzonis Einschätzung, dass Patrizis synthetische Konzeption des poetischen Furors bisher nicht differenziert genug betrachtet und stets als platonisch eingeordnet wurde. Siehe Bolzoni, L'universo, S. 17.

74 Was also als Begeisterung bezeichnet wird, scheint eine Mischung zweier Bewegungen zu sein. Die eine, die die Seele ertragen muss, die andere, aufgrund der sie sich gemäss ihrer Natur bewegt.

75 Patrizi zitiert Plutarch, De Pythiae oraculis, 404e.

76 Zwei Bedingungen müssen vorhanden sein, jene des (genialen) Verstandes und jene des Furors, um den Dichter perfekt zu machen.

77 Bolzoni, L'universo, S. 32.

78 Die Dreiteilung in Gott, Natur und Kunst wirkt sich auch auf die Unterteilung der Genres aus. Vom Furor geprägt sind die Genres des Prophetischen, des Lobgedichts (« encomiastico ), des Lehrgedichts und des Märchens, von der Natur geprägt sind die Genres des Schmerzhaften, des Fröhlichen, des Verächtlichen und des Burlesken ( $D A$, II, 283-284). Matuschek warf Patrizi vor, er habe eine «Säkularisierung des Staunens » herbeigeführt und das Staunen «vom prätentiöseste[n] [...] zum trivialsten Blick des weltlichen Staunens » verkommen lassen - und verkennt dabei, dass der Dichter wie auch seine Inhalte in der Konzeption Patrizis immer auch Anteil am Göttlichen haben. Zum einen wird der Dichter an die göttliche Inspiration zurückgebunden, zum anderen gelten jene Dichtungen als die ehrwürdigsten, die von der göttlichen Ewigkeit künden und zeugen. Siehe Matusche K, Über das Staunen, S. 137, 147. 
Concioscia cosa che, se bene egli non è Dio, tiene almeno del divino, poscia che i primi e più fini poeti per divina spirazione poetarono e a poetare seguitarono molti anni. E s'egli non è natura, molti del suo lignaggio furono da natura a poetar portati. E s'egli non è artefice, molte cose molti poeti hanno per arte fatte. ${ }^{79}$ $(D A, \mathrm{II}, 285)^{80}$

In dieser aussergewöhnlichen Rolle erwächst dem Dichter die Fähigkeit, Neues zu kreieren (DA, II, 286). So haben Dichter wie Linos, Orpheus, Parmenides und Empedokles in ihren Theogonien und Kosmogonien ganze Welten, ja sogar die Götter und die Titanen erschaffen (DA, II, 287).

Conciosia cosa che chi altro che il poeta creò dopo Dio questo mondo? Lino, ed Orfeo, e Pronatide, e Parmenide, ed Empedocle nelle loro Cosmogonie, e Cosmopoie e Protocosmi! Chi creò tutti i Dei, altri che il poeta Orfeo [...]? Chi creò i Titani di nulla, altro che i poeti Tamira, Eumolpo [...]? [...] E create son di nulla molte parti ne' poemi, sì come è la lancia d'Argaglia, il corno di ... e l'impazzir di Orlando e simili altre cose nuove, da niune per adietro poetate. ${ }^{81}$ (DA, II, 287)

Was der Dichter in seiner Funktion als schöpferischem « emolo » Gottes, der Natur und des Künstlers schafft (DA, II, 289), ist das Wunderbare, das bei den Zuhörern und Lesern Verwunderung und Staunen auslöst:

La prima [facitura] tra le quali sia che mirabile e maraviglioso da noi si chiama

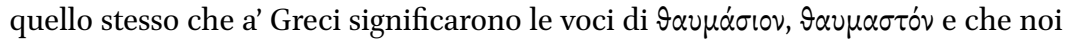
chiamiamo ancora ammirabile, e ammirando e maraviglioso. E quanto è all'opera che egli fa, mirabile appelliamo quello che di sua natura è atto a commuovere meraviglia in chi l'ascolta, o vede, o legge, o d'altra guisa sente, o intende $[\ldots]^{82}$ $(D A$, II, 290)

79 Es ist also so, dass er, auch wenn er nicht Gott ist, Göttliches in sich trägt, sodass die ersten und die vollendetsten Dichter aufgrund göttlicher Inspiration dichteten und viele Jahre damit fortfuhren. Und wenn er nicht Natur ist, so wurden doch viele natürlicherweise durch seine Sprache zur Dichtung gebracht. Und wenn er nicht Künstler ist, so haben doch viele Dichter vieles mit Kunstfertigkeit gemacht.

8o Weinberg, A History, S. 773: «The poet is not God, but he has something of the divine; he is not Nature, but he is made to poetize by Nature; he is not an artificer, but he does many things by art. Hence, the very way in which he creates is marvelous [...] ».

81 Wer sonst als der Dichter hat nach Gott diese Welt erschaffen? Linos und Orpheus und Pronatides und Parmenides, und Empedokles in ihren Kosmogonien und Cosmopoie und Protokosmen! Wer schuf alle die Götter, wer anderes als Orpheus [...]? Wer schuf die Titanen aus dem Nichts, wer anderes als die Dichter Tamira, Eumolpos [...]? Und aus dem Nichts wurden viele Elemente der Dichtung geschaffen, so wie die Lanze von Argaglia, das Horn von ... und die Raserei von Orlando und weitere ähnliche Neuheiten, von denen nie zuvor gedichtet wurde.

82 Das erste unter den Elementen, das wir wunderbar und erstaunlich nennen, ist das-

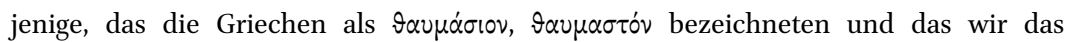
Bewundernswerte, Verwunderliche und Erstaunliche nennen. Und was das Werk betrtifft, 
Das Wunderbare und Erstaunliche ist das, das in den Zuhörern und Lesern Staunen auslöst. Es wird damit in erster Linie über seine Wirkung, nicht über seinen Inhalt oder seine Beschaffenheit definiert.

\subsubsection{Der Ursprung der Poesie}

Nicht die Nachahmung ist der Ursprung der Dichtung, sondern die Dichtung ist der Ursprung der Nachahmung.

Adunque non la imitazione partorì la poesia, ma la poesia generò in se medesima e nel suo ventre la poetica imitazione. ${ }^{83}(D D$, II, 41$)$

Die Dichtung nahm ihren Anfang, als der Mensch anfing zu singen: «[...] Gesang nicht nur der Stimme, wie ihn viele Vögel und Menschen oft anstimmen, sondern Gesang der Worte » $(D I, I, 9)$. Da diese Anfänge nicht belegt sind, greift Patrizi auf die erste Erwähnung eines Sängers zurück - und diese findet sich in der Bibel (Gen., 4, 21). Jubal, ein Bruder Noahs, sei der Vater der « cantanti in citara », der Zithermusiker bzw. -sänger gewesen. Die Zither («cetra») ist nicht nur ein Instrument, sondern gleichsam das Symbol der poetischen Eingebung und Gabe. ${ }^{84}$ Von Jubal führt Patrizi die Entstehung der Dichtung über Noah und dessen Söhne hin zu Zarathustra (Mesopotamien) und Osiris (Ägypten), wobei historische und mythische Belege aus verschiedenen Kulturen zusehends verwoben werden. So habe Osiris in seiner Armee jungfräuliche Musikerinnen mitgeführt, welche die Musen mit ihrem Anführer Apollon gewesen seien. Platon schliesslich erwähnt in seinen Nomoi Isis - die Gemahlin von Osiris als göttliche Dichterin $(D I, I, 11)$. Patrizi führt noch einige weitere mythische Belege an - aus dem Alten Testament, aus Mesopotamien, Libyen, Arabien -, wobei er diese mit dem Hinweis auf die « oscurità di tanta antichità » in eine Sphäre des Geheimnisvollen und Enigmatischen hüllt. ${ }^{85}$

In der Deca istoriale werden weitere Belege aufgelistet und ausgeführt, so zu Phemonoe - erste Priesterin und Prophetin des delphischen Orakels sowie Tochter von Apollon ${ }^{86}$ - und Olenos, den Herodot vor Linos, Panfo und Orpheus

das er hervorbrachte, bezeichnen wir dasjenige als wunderbar, das von seiner Natur aus Erstaunen in demjenigen hervorruft, der es hört oder sieht oder liest oder auf andere Weise mitbekommt oder vernimmt.

83 Es hat also nicht die Nachahmung die Dichtung hervorgebracht, sondern die Dichtung hat in sich selber, in ihrem Bauch, die poetische Nachahmung gezeugt.

84 Vgl. Petrarca, Canzoniere, edizione commentata a cura di Marco Santagata, Milano 1996, S. 1158, Sonnett CCXCII, Vv. 13-14.

85 Wir erinnern uns an das enimma als Eigenschaft der Dichtung (DA, II, 255).

86 Auf Phemonoe hat bereits Vida verwiesen: Sie sei die Erste gewesen, die das ursprüngliche Metrum des Hexameters verwendet habe. Siehe De arte poetica, I, Vv. 33-43. 
als ersten (Hymnen-)Dichter nennt. Die Deca ammirabile aber setzt direkt bei Orpheus ein, denn zu Orpheus' Attributen gehört es - wie bereits mehrfach gesehen -, nicht nur die Menschen, sondern auch Vögel, Fische, wilde Tiere, Wälder, Berge, Flüsse und Lüfte in Staunen zu versetzen $\left(D A\right.$, II, 257) ${ }^{87}$ Mit Orpheus wird somit ein direkter Bogen zu den zentralen Themen des mirabile und der maraviglia geschlagen, sind die beiden Elemente in seinen Gesängen doch paradigmatischerweise miteinander verknüpft.

Alla quale [qualità] ed esse tutte come ad un capo si riducano, e tutta la poesia indi penda, e forma prenda e vita. E questa qualità noi chiamiamo col nome di mirabile. E mirabile intendiamo che sia tutto quello che in altrui o muove, od è a muovere atto maraviglia. ${ }^{88}(D A, \mathrm{II}, 257)$

Zudem werden am Beispiel des Orpheus die verschiedenen Begriffsnuancen des Staunens erläutert: Nebst dem eher neutralen maravigliare führt Patrizi einen weiteren Begriff ins Feld: « $\chi \eta \lambda \eta \dot{ } \sigma \omega »$, mit der Bedeutung von « con la sua forza incantare per istupore » $\left(D A, \mathrm{II}, 25^{8}\right)$. Patrizi leitet diese Bedeutung aus den Orphischen Argonautika ${ }^{89}$ ab und fügt sogleich an, worin sich stupore und maraviglia unterscheiden:

E lo stupore è una forte maraviglia, come in proseguendo si scoprirà palese. Dunque il canto, perchè fa maravigliar altrui, meritamente sarà da tenersi per mirabile..$^{90}(D A$, II, 258$)$

Genauso wie stupore wird auch « हैं $\kappa \tau \tau \alpha \sigma l \nu »$, angelehnt an Pseudo-Longin, als « grosses und starkes Staunen » beschrieben ( "grande e forte maraviglia », $D A$, II, 259-26o). Damit wird die maraviglia zusätzlich mit den Bedeutungen des Magischen und des Aussersichseins konnotiert.

Die ältesten und ursprünglichsten Texte waren poetische Texte: «Alle Wissenschaften, von den göttlichen, den natürlichen oder den menschlichen Dingen, wurden als Dichtung geschrieben $(D A, \mathrm{II}, 238)$. Die ersten Poeten

87 Siehe $D A$, II, 257; ebenso $D D$, II, 144. Vgl. dazu auch den Anfang der Silva Manto von Poliziano.

88 Zu dieser Qualität reduzieren sich alle wie zu einem Haupt, und die ganze Dichtung hängt davon ab und nimmt Form und Leben an. Und dieser Qualität geben wir den Namen des Wunderbaren. Und als Wunderbares meinen wir all das, was in anderen Erstaunen hervorruft oder fähig ist, die Bewegung des Staunens hervorzubringen.

89 Francis Vian (Hg.), Les argonautiques orphiques, texte et traduction, Paris 1987, S. 79, Vv. 73-74.

9o Und die Verblüffung ist ein starkes Staunen, wie es sich im Folgenden klar zeigen wird. Also ist der Gesang, weil er andere zum Staunen bringt, verdienterweise als wunderbar zu definieren. 
waren «Väter und Anführer der Weisheit » und somit die Dichtung Ursprung allen Wissens und aller Weisheit. Aber nicht nur über die wirklichen und wahren Dinge wurde gedichtet, genauso oft wurden Geschichten erfunden, Fiktionen produziert (DA, II, 239). Die ältesten Mythen waren aus mirabilia zusammengesetzt: «das heisst aus Neuheiten, Paradoxen, übernatürlichen Dingen und Inhalten, die über das Gewohnte hinausgehen » (DA, II, 265-266). Der staunenswerte Charakter der Dichtung sowie die staunende Inklination der Zuhörer und Leser gehören somit seit Anbeginn der Kultur zur Dichtung.

\subsubsection{Das Wunderbare der Dichtung}

Das Wunderbare der Dichtung manifestiert sich in allen ihren Bestandteilen und ist auf allen Ebenen präsent:

[...] così doverà il mirabile non / in questa, $\mathrm{o}$ in quella, parte del poema mandare i raggi suoi, ma diffondergli per tutte, siasi egli fatto da poeta o come creatore, o, come è natura, generatore, o, come è huom artista, facitore. ${ }^{91}(D A$, II, 329)

$[\ldots]$ il poeta $[\ldots]$ doverà per tutte le parti sue [della poesia] fare il mirabile risplendere. ${ }^{92}(D A, \mathrm{II}, 329-330)$

[...] il soprano grado di poetica perfezione sta riposto nella composizione sudetta del mirabile, [...] sparso per tutte le membra del poema; e questo essere la compiutissima forma sua, e universale, e commune a tutta la poesia $[\ldots]^{93}$ $(D A$, II, 330)

Analog zur Vernunft, die Patrizi als ontologische Form und Essenz des Menschen begreift, sieht er im mirabile das Wesen der Dichtung begründet $(D A, \mathrm{II}, 332)$. Aus diesem aristotelischen Formverständnis leitet sich sogleich auch das Ziel ab, auf das der Dichter sich ausrichten soll. ${ }^{94}$ Weder Platon, Aristoteles noch Horaz haben das Ziel der Dichtung in dieser Weise definiert: Sie fokussierten auf die gesellschaftliche, politische und didaktische Funktion der Dichtung, auf deren erfreuliche oder aber reinigende Wirkung in Bezug auf Affekte und Leidenschaften oder aber sahen das einzige Ziel der Dichtung

$91[\ldots]$ so soll das Wunderbare seine Strahlen nicht / in diesen oder in jenen Teil des Gedichtes aussenden, sondern überall ausbreiten, sei jenes vom Dichter als Schöpfer geschaffen oder nach der Natur gebildet oder vom Künstler gemacht.

$92[\ldots]$ der Dichter [...] muss das Wunderbare in allen Teilen [der Dichtung] zum Erstrahlen bringen.

$93[$ [...] der höchste Grad dichterischer Perfektion zeigt sich in der Komposition des Wunderbaren, [...] verteilt in allen Gliedern des Gedichtes; und das sei die vollendete und universelle Form, die allen Dichtungen gemein ist [...]

Vgl. Bolzoni, L'universo, S. 136. 
in der Mimesis ( $D A$, II, 333). Aus der Uneinigkeit über das effektive Ziel der Dichtung - Patrizi führt auf mehreren Seiten einen ganzen Katalog von Zielen auf $(D A$, II, 342) - sowie der Unterschiedlichkeit der Dichtungen folgert Patrizi, dass die bisher bekannten Wirkungskategorien zwar nicht falsch, aber nicht universell gültig seien.

Adunque egli è da ricercarne uno che due condizioni habbia sopra tutti: l'una che sia proprio della poesia e non commune ad altre scritture, l'altro che sia generale a tutta, sì che niuna non sia che di suo uffizio a quel fine non sia indirizzata [...] Ora questo fine così fatto a noi non pare che esser possa altro che quello che dicemmo dinanzi, e che dalla diffinizione essenziale nasce del poeta e della poesia. E ciò si è il mirabile per fine universale intrinseco, che forma anco si chiama, e la maraviglia per fine estrinseco. ${ }^{95}(D A, \mathrm{II}, 343)$

Diese Bestimmung des mirabile als intrinsische Qualität der Dichtung und der maraviglia als extrinsisches Wirkungsziel gehört zu den Kernsätzen von Patrizis Poetik.

Der abstrakten Formulierung entspricht ein Katalog mit konkreten Merkmalen, die die intrinsische Qualität des mirabile herbeiführen und im poetischen Text verankern. Das Vorgehen ist analog zum bereits beschriebenen: Zuerst erfolgt eine umfangreiche Auflistung verschiedener Beschaffenheitsmerkmale des poetischen Textes, die seit der Antike überliefert wurden. Diese werden geprüft - entweder verworfen oder beibehalten -, bis ein reduzierter Katalog feststeht.

[...] entusiasmo, profezia, enimma, sapienza, favola, allegoria, varietà, aggrandimento, sminuzzamento, evidenza, dolcezza, lingua straniera, verso, canto. Delle quali alcune stanno ne' poemi come cagioni, altri come materie, e alcune come modi del trattarle, e altre come stromenti, e per tanto distinguianle anco per queste vie. ${ }^{96}(D A$, II, 248)

95 Wir müssen also jene Eigenschaft finden, die in erster Linie zwei Bedingungen erfüllt: Die erste ist, dass sie nur der Dichtung und keinen anderen Schriften eigen ist, die andere, dass sie jeder Dichtung zukommt, sodass es keine gibt, die sich der Pflicht, auf jenes Ziel ausgerichtet zu sein, entzieht [...] Dieses so gestaltete Ziel scheint kein anderes sein zu können als jenes, von dem wir bereits gesprochen haben, und das aus der essentiellen Definition des Dichters und der Dichtung hervorgeht. Und das ist das Wunderbare als universelles und intrinsisches Ziel, das wir auch als Form bezeichnen, und das Staunen als extrinsisches Ziel.

96 [...] Begeisterung, Weissagung, Enigma, Weisheit, Erzählung, Allegorie, Vielfältigkeit, Vergrösserung, Verkleinerung, Evidenz, Sanftheit, verfremdete Sprache, Vers, Gesang. Von diesen Eigenschaften sind einige in der Dichtung als Ursachen, andere als Stoffe, weitere als deren Bearbeitungstechniken, einige als Instrumente; und nach diesen Ausrichtungen sind sie auch zu unterscheiden. 
Allen voran steht der Enthusiasmus, der den Kreationsprozess überhaupt erst auslöst. Weitere Ursachen («cagioni ») sind Freude, Schmerz, Empörung, Humor sowie die Kunstfertigkeit. Patrizi gliedert die cagioni wie folgt: «Furor und Kunstfertigkeit und in der Mitte die Natur, die vier Affekte (Schmerz, Freude, Empörung und Spass) / beinhaltet» (DA, II, 249).

Als Stoffe («materie ») bezeichnet Patrizi die Weissagung, die Weisheit, die Erzählung ${ }^{97}$ sowie die Allegorie. Die Stoffe können wahr, für wahr gehalten oder aber fiktiv sein. Zu den Modi («modi ») zählen das Enigma, die Vielfalt, die Vergrösserung, die Verkleinerung, die Evidenz sowie die Sanftheit. Während es die Evidenz nur für bestimmte Beschreibungen und die Verkleinerung nur in einzelnen Genres braucht, kommen die anderen Modi in allen Genres und Subgenres vor. Zu den sprachlichen Instrumenten («stromenti ») zählen die «verfremdete Sprache, Vers und Gesang » - alle drei Elemente finden sich in allen antiken Dichtungen.

Das mirabile ist die Verdichtung der genannten Qualitäten zu einem unverwechselbaren Ganzen: «Zu dieser Qualität reduzieren sich alle wie zu einem Haupt, und die ganze Dichtung hängt davon ab und nimmt Form und Leben an. » $(D A$, II, 257)

Der Enthusiasmus («entusiasmo») erzeugt Staunen, weil er unberechenbar ist und nur selten auftritt, weil er dem Göttlichen entstammt und göttliche Werke hervorbringt. Dass der Enthusiasmus bei den Qualitäten des mirabile aufgeführt ist, bedeutet, dass er als Stimmung oder Prägung in die Dichtung einfliesst und für die Leser wahrnehmbar wird (DA, II, 261). ${ }^{98}$

Die Weissagung («profezia») produziert Staunen, weil der Blick in die Zukunft göttlicher und enigmatischer Natur (« enimma ») ist.

[...] la profezia, originaria forma di composizione poematica ispirata dal sacer furor divino, era enigmatica, oscura $[\ldots]^{99}$

Das Enigma ist wunderbar und löst Staunen bei den Zuhörern und Lesern aus, weil es rätselhaft und das Rätselhafte mit dem Staunenswerten verschwistert ist (DA, II, 26o). Das Enigma zeichnet sich dadurch aus, dass es weder völlig undeutlich noch eindeutig klar ist, sondern zwischen diesen beiden Polen changiert. Es war bereits Teil der frühen Prophezeiungen und Orakel und es haftet ihm von jeher das Wundersame an.

97 «Favola» müsste richtigerweise als „fiktive Erzählung“ wiedergegeben werden, denn Patrizi versteht darunter in erster Linie die Fiktion. Der Einfachheit halber verwende ich nur den Begriff „Erzählung“.

98 HathaWAY, Marvels, S. 66.

99 Rinaldi, $I l$ «Trimerone », S. 28. 
Die Weisheit («sapienza») der antiken Autoren wird seit jeher bewundert. Zur sapienza gehören folgende vier Bereiche: Wissen(-schaft), Kunst, Moral und Geschichte. Dass das Staunen mit Wissen verbunden ist, führt Patrizi auf Platon und Aristoteles zurück, die beide das Staunen als Ausgang des Philosophierens definiert haben. ${ }^{100}$ Alles, was in der Natur vorkommt und dessen Ursache wir nicht kennen, bestaunen und bewundern wir. Zudem schreibt Aristoteles in der Nikomachischen Ethik, dass allen Erscheinungen der Natur das Göttliche beiwohne und dieses Göttliche Staunen erzeuge (DA, II, 262). Die Kunst erzeugt Staunen, weil sie es den Menschen ermöglicht, Werke ausserhalb des Natürlichen herzustellen. In der Dichtung enthaltene Moral ist staunens- und bewundernswert, da - laut dem Mythos, den wir schon bei früheren Autoren wie Poliziano finden - die Dichtung die Menschen zivilisiert und ihnen die Regeln für die Gemeinschaft gelehrt hat. Die Geschichte ist immer staunenswert, wenn sie poetisch verarbeitet wird - wobei es Aufgabe der Poeten ist, spannende und ausserordentliche Elemente aus der Geschichte herauszugreifen und sie erstaunlich darzustellen.

Die Erzählung («favola») setzt sich aus Staunenswertem zusammen, wofür Patrizi auf die berühmte Aristoteles-Passage zu Beginn der Metaphysik rekurriert:

(So ist auch ein Liebhaber von Mythen in gewisser Hinsicht ein Philosoph, setzt sich doch ein Mythos aus Wunderbarem zusammen. $)^{101}$

Konkrete Charakteristika der Erzählung sind für Patrizi die Fiktion, das Neue und das Paradoxe. Er unterstreicht dies mit einem einschlägigen Zitat Plutarchs:

«e in somma appo lui [Omero] è un racconto di cose paradosso e favoloso, per empiere di agonia e di maraviglia i lettori e fare un ascoltamento pieno di stupore $»^{102}(D A, \text { II, 264 })^{103}$

Ebenso gehört dazu, dass in der Erzählung Dinge beschrieben werden können, die die Natur übersteigen.

\footnotetext{
$100 \quad$ Met., 982b, Tht., 155d, eth. Nic., 1153 b.

101 Met., 982b.

102 «schliesslich ist es gemäss ihm [Homer] eine Erzählung mit paradoxen und märchenhaften Inhalten, um die Leser mit Agonie und Staunen zu erfüllen und das Zuhören mit Verblüffung zu versehen »

103 Patrizi nennt Plutarch als Quelle, tatsächlich handelt es sich um Pseudo-Plutarch: De vita et poesi Homeri, II, vi.
} 
La favola, adunque, in genere e la favolosa poesia più antica di mirabili fu composta; ciò è di novità, di paradossi, di cose superanti la natura, di cose inalzate sopra il consueto. ${ }^{104}\left(D A, \mathrm{II}, 265^{-266)}\right.$

Die Allegorie («allegoria») bezeichnet Patrizi als eine Art Enigma, weil unter ihrer Oberfläche («scorza») unerwartete Bilder und Ideen, wenn nicht gar verborgene Wahrheiten zum Vorschein kommen. Der Vielfalt und der dichterischen Fantasie für neue Allegorien sind quasi keine Grenzen gesetzt (DA, II, 266) und die Fantasie führt, so lehrte es bereits Pseudo-Longin zum Staunen bzw. zur Erschütterung $\left(D A\right.$, II, 267) ${ }^{105}$ Patrizi spricht explizit auch den bildlichen Charakter der Allegorie an, der Schönes und Kostbares in sich birgt und zum Staunen führt, wenn er entdeckt wird.

Vielfalt und Vergrösserung («varietà, aggrandimento ») sind Merkmale des mirabile, insofern als die Leser über die vielfältigen Inhalte und Ausprägungen der Dichtung sowie deren Nähe zum Göttlichen staunen. Die Grösse hebt die Dichtung über die Realität und die Natur hinaus. Die minutiöse Beschreibung und die Evidenz («sminuzzamento, evidenza ») tragen zum Staunen bei, weil sie vor dem inneren Auge des Zuhörers Bilder erscheinen lassen, die ausserhalb seines gewohnten Blickfeldes liegen. Die Bilder machen den Zuhörer und Leser zu einem aktiv und kreativ Beteiligten; seine Imagination muss in die Lektüre einfliessen, damit der entsprechende Effekt der maraviglia sich einstellt.

Auf sprachlicher Ebene kommt dem Vers besonderes Augenmerk zu, wobei Patrizi auf Ludovico Castelvetro, dessen aristotelische Sichtweise er sonst scharf kritisiert, zurückgreift. Er zitiert aus dessen Kommentar der aristotelischen Poetik:

« Alla quale s'aggiunge il verso, che è parlare maraviglioso e dilettevole per molte cagioni. » $[\ldots]$

«Ed è cosa ragionevole che Dio parli in molto più eccellente modo, che non parlano comunemente gli huomini. » $[\ldots]$

\footnotetext{
104 Die Erzählung im Allgemeinen, wie schon die älteste erzählende Dichtung, ist aus Wundern zusammengesetzt; das heisst aus Neuheiten, Paradoxen, übernatürlichen Dingen und Inhalten, die über das Gewohnte hinausgehen.

105 Sublim., 15, 2. Pseudo-Longin benutzt « ekplexis », Patrizi übersetzt mit «stupore ».
} 
«E massimamente veggendo che i risposi divini di Apollo erano dati in così fatti versi $[\ldots] »^{106}\left(D A, \mathrm{II}, 25^{8}\right)^{107}$

Castelvetro führt den Vers auf das Sprechen der Götter sowie die Differenz zur Alltagssprache zurück - letztere bezeichnet Patrizi als lingua straniera. Beides - das Göttliche wie das Abweichen vom Gewöhnlichen - ist erstaunlich und erfreulich («maraviglioso e dilettevole»), weil es Neues, Unerwartetes, Fremdes hervorbringt.

Die Sanftheit («dolcezza ») findet sich in allen Bereichen des Gedichts, vor allem aber im Gesang und in der Harmonie der Verse. Sie soll - mit Patrizi bildlich gesprochen - die Dichtungen wie Puderzucker überziehen. Sie trägt massgeblich nicht nur zum Staunen, sondern auch zum Vergnügen (« diletto ») bei $(D A$, II, 259).

Patrizi führt bewusst Kriterien der inventio, der dispositio sowie der elocutio zusammen auf, da er Dichtung nicht als die Summe dieser einzelnen Teile, sondern als Ganzes betrachtet. Die Kriterien sind zudem nur sehr generisch spezifiziert; es sind keine Vorschriften, sondern Eckwerte, anhand derer Erstaunliches entstehen kann. Ganz eindeutig legen die Beschreibungen ihren Fokus auf die Wirkung - die Qualität des mirabile entsteht nicht (nur) aufgrund der Beschaffenheit des Textes, sondern erst, wenn dieser seine Wirkung auf den Zuhörer und Leser entfaltet. Insofern dieses Ziel erreicht wird bzw. erreicht werden will, sind der dichterischen Fantasie kaum Grenzen setzt. Patrizi stellt keine Liste bevorzugter Genres auf, tendiert aber zumindest implizit zu offenen Formen der Lyrik und des romanzo, während er die strengen Formen der Tragödie und des poema eroico als wenig reizvoll erachtet. ${ }^{108}$ Er gibt auch keine stilistischen Vorgaben - einzig die Versform ist gegeben, da diese seit jeher das Göttliche der Dichtung repräsentiert.

\subsubsection{Der staunende Rezipient}

Die fundamentale Neuerung, die Patrizi in die literaturtheoretische Diskussion seiner Zeit einbringt, ist der Fokus auf die „Psychologie“ des Lesers.

106 «Zu dieser kommt der Vers hinzu, der erstaunliches und vergnügliches Sprechen aus vielerlei Hinsicht ist. » [...] «Und es ist eine berechtigte Annahme, dass Gott in einer sehr viel herausragenderen Weise sprach, als es die Menschen gewöhnlich tun.» [...] «Und vor allem sehen wir, dass die göttlichen Antworten des Apollon in derart verfassten Versen gegeben wurden [...]»

107 Patrizi zitiert aus Lodovico Castelvetro, La poetica di Aristotele vulgarizzata, et sposta, Wien 1570 .

108 HathaWAY, Marvels, S. 68. 
Dieser findet sich im zehnten Buch der Deca ammirabile und bildet die Klimax der gesamten Deca.

Patrizi versucht, die maraviglia bzw. ihre Funktionsweise und ihren Ort in der Seele des Menschen zu bestimmen. Das Staunen manifestiert sich als eine Art des Ergriffenseins des Geistes und bisweilen auch des Körpers, wobei Patrizi dieses Ergriffensein nicht als Eigenschaft, sondern als Bewegung fasst. Diese vollzieht sich primär innerlich, kann sich aber auch äusserlich manifestieren. Wie schon in seiner Bestimmung des Dichters und der Dichtung leitet er die Definition der Staunensbewegung aus der Auseinandersetzung mit etablierten Begriffen und Konzepten ab:

Das Staunen funktioniert weder vegetativ noch sinnlich oder diskursiv. Es ist nicht vegetativ, weil es nichts zur Ernährung und Erhaltung unseres Körpers beiträgt. Es ist nicht sinnlich, weil es keinem der Sinne zugeordnet werden kann. Die Sinne staunen nicht, aber Sinneseindrücke können das Staunen herbeiführen oder dieses begleiten. Es ist nicht diskursiv, weil es sich nicht in Worten ausdrückt, weil es keine Begriffe hervorbringt, bestätigt oder negiert (DA, II, 355). Das Staunen ist auch dem Intellekt nicht zuzuordnen, denn dieser ist die Basis unseres Wissens und das Staunen resultiert - zumindest zu einem gewissen Teil - aus dem Nichtwissen (DA, II, 355-356). Aufgrund der Zeichen, die Staunende nach aussen senden, über das Gesicht - hochgezogene Augenbrauen -, die Augen - weit geöffnete Augen - und den Körper, könnte es scheinen, dass das Staunen ein Affekt sei, analog zur Liebe, zum Hass, zur Wut und zu den anderen Gefühlsregungen. Da das Staunen aber gleichzeitig der Auslöser dieser körperlichen Reaktionen ist, scheint die Zuteilung zu den Affekten nicht adäquat. Und während die Affekte einen spürbaren entweder erfreulichen oder trübenden, ja gar schädigenden Effekt auf uns haben können, kann das Staunen auch ohne Emotionen auskommen ( $D A$, II, 357). Patrizi veranschaulicht, dass die Begleit- oder Folgeerscheinungen des Staunens - wie Mitleid, Wut oder Liebe - wieder verebben können, während die maraviglia anhält: Wir staunen und wundern uns darüber, dass ein Freund uns betrogen hat, es folgen Wut und Rachegelüste. Haben wir Rache geübt, flacht die Wut ab, während das Staunen weiterhin anhält ( $D A, \mathrm{II}, 357)$. Weiter sind die Affekte im « ungestümen und sinnlichen / Teil der Seele» (DA, II, 358) angesiedelt, während das Staunen diesen - wie auch den kognitiven - Bereich nur tangiert.

Die Koppelung an den kognitiven Bereich wird folgendermassen hergeleitet: Wer gar nichts weiss, einen Sachverhalt nicht kennt und keine Begriffe - von Schönheit beispielsweise - hat, der kann weder Entsprechung, Abweichung noch Übersteigung erkennen und somit auch nicht erstaunt sein $(D A$, II, 358). Patrizi schliesst daraus, dass das Staunen aus der kognitiven Fakultät hervorgeht und auf die affektive Fakultät einwirkt - es fliesst als Bewegung 
zwischen diesen Bereichen hin und her. Er geht noch weiter und bezeichnet die maraviglia nicht einfach als Bindeglied, sondern schafft neben der « erkennenden Potenz» («potenza atta a conoscere») und der «affektiven Potenz» («potenza atta ad affenzionarsi») eine eigenständige «staunende Potenz » («potenza atta a maravigliarsi », $\left.D A, \mathrm{II}, 35^{8}\right)$.

E di più èla maraviglia commune alle conoscenti potenze e alle affettuose, e corre per tutte quelle, e per tutte queste. Adunque ella sarà una potenza mezzana tra quelle due, e il moto suo un terzo moto in mezzo a quelli per tutti loro, dal mezzo all'alto, e dal mezzo al basso discorrente. Il qual moto, dicendosi ammirazione, dare dovrà il nome alla potenza sua ammirativa $[\ldots]^{109}(D A$, II, 361$)$

Dass die potenza atta a maravigliarsi zur potenza ammirativa («bewundernden Potenz », $D A$, II, 360) umbenannt wird, rührt daher, dass es von maraviglia keine aktive adjektivische Form gibt, welche die Bewegung des Geistes wiedergeben könnte. Maraviglioso mit der Bedeutung von „wunderbar, erstaunlich“ ist auf das Objekt bezogen, nicht auf das staunende Subjekt. Da es hier aber um ebendieses staunende Subjekt geht, wird die Potenz ammirativa genannt; ihre Bewegung ist die maraviglia.

E questi uffici [ufficio di mente e ufficio di affetti] fu acconcia a fare la ammirativa, posta in mezzo a due contrari, per via della maraviglia, moto ed atto suo. ${ }^{110}$ $(D A, \mathrm{II}, 361)$

Die Neuheit dieser Erkenntnis ist Patrizi durchaus bewusst:

Ma niuno de' filosofanti disse mai tra conoscente potenza e affettuosa trovarsene di mezzo una terza. ${ }^{111}\left(D A\right.$, II, $\left.35^{8}\right)$

Patrizi führt weiter aus, wo dieser Bereich der potenza ammirativa angesiedelt sein könnte. Die Bewegung des Staunens kann nicht mit den Sinnen oder mit dem sensus communis, der über die Sinneseindrücke Objekte erkennt und

109 Zudem ist das Staunen der erkennenden und der affektiven Potenz gemeint und fliesst durch diese beiden. So ist sie eine mittlere Potenz zwischen diesen beiden, und ihre Bewegung eine dritte Bewegung inmitten von diesen, von der Mitte nach oben und von der Mitte nach unten fliessend. Diese Bewegung, nennen wir sie Bewunderung, gibt der bewundernden Potenz den Namen [...]

110 Und diese Aufgaben [Aufgabe des Geistes und Aufgabe der Affekte] waren geeignet, um die bewundernde Potenz in der Mitte zweier Gegensätze über den Weg des Staunens zu ihrer Bewegung und Geste zu machen.

111 Aber keiner der Philosophen hat je gesagt, dass sich zwischen der erkennenden und der affektiven Potenz eine dritte in deren Mitte befinde. 
unterscheidet, identifiziert werden, denn weder staunen die Sinne, noch trägt das Staunen zur Qualifikation der Objekte bei. Auch mit der Fantasie kann sie nicht gleichgesetzt werden, denn sie ist keine Bewegung der Fantasie, sondern höchstens eine Folge davon. Die Fantasie hat laut Patrizi ihren bestimmten Ort innerhalb der potenza conoscitiva, sie kann diesen Ort aber nicht überschreiten und - wie das Staunen - bis in den diskursiven Bereich gelangen $(D A$, II, 360$) .{ }^{112}$

La merveille n'est pas un acte ou un mouvement de l'imagination. Elle ne relève pas non plus de l'opinion, ni du discours. En fait, elle découle de toutes les puissances associées à la connaissance. Pour mieux dire, elle précède leurs mouvements, les accompagne et les suit. ${ }^{113}$

Von zentraler Bedeutung ist der verbindende Charakter der maraviglia. Erkennender Geist und Affekt hätten keine Möglichkeit, miteinander zu kommunizieren - sie sind kategorial unterschieden -, wenn nicht die maraviglia ebendiese Kommunikation zwischen ihnen herstellen würde (DA, II, 361). Die potenza affettuosa richtet sich auf körperliche Begehren und Animositäten, während die potenza conoscitiva auf Besonnenheit, Wissen und Weisheit ausgerichtet ist - die potenza ammirativa verbindet beides und, spinnt man den Gedanken weiter, macht den Menschen erst zu dem, was er eigentlich ist.

Onde si fa palese che l'ammirazione è mezzano stormento dell'elezione dell'una vita e dell'altra. E non si truoverà huom nessuno, il quale dall'una all'altra vita sia passato /, o per passare sia, se non per lo mezzo della maraviglia, presa più di quella a cui si passa, che dell'altra onde si parte. ${ }^{114}\left(D A, \mathrm{II},{ }_{362}\right)$

Patrizi verwendet als Bild die Meerenge von Euripos, durch die das Meer ein- und ausströmt: Das Meer ist die Bewegung der maraviglia, die zwischen

112 Die Imaginationsfähigkeit der Rezipienten gehört als Teil zur potenza conoscitiva, wird aber nicht weiter kommentiert. Auch in Bezug auf den Poeten geht Patrizi nicht speziell auf die Fantasie ein; er nennt quasi beiläufig, dass es zwei unterschiedliche Arten von Künstlern gibt: die einen, die nachahmen, und die anderen, die ihrer Fantasie Ausdruck verleihen - wobei er Letzteres favorisiert ( $D D$, II, 88) Patrizi bringt auch die inventio nicht mit der Imaginationsfähigkeit des Poeten in Verbindung. Vgl. HathawaY, Marvels, S. 68.

113 BÉLANGER, Bomarzo, S. 247.

114 Damit wird deutlich, dass die Bewunderung ein verbindendes Instrument bei der Wahl des einen oder des anderen Lebens ist. Und es wird sich kein Mensch finden, der vom einen ins andere Leben hinübergegangen ist / oder der hinübergehen wird ohne das Mittel des Staunens, stärker angezogen von dem, auf das er zugeht, als von dem, das er zurücklässt. 
der Kognition und den Affekten hin- und herströmt. ${ }^{115}$ Dadurch, dass die maraviglia zur Trägerin affektiver und kognitiver Strömungen werden kann, kann sie alle Bereiche der menschlichen Seele (anima) durchdringen.

Noch ungeklärt ist die Frage, ob alle oder nur gewisse Menschen staunen können. Grundsätzlich meint Patrizi: « Wir glauben, dass alle Menschen fähig sind, vom Staunen erfasst zu werden » («Tutti gli huomini crediamo noi essere atti ad essere presi da maraviglia [...]»). Lediglich zwei Ausnahmen gebe es: zum einen die vollkommen Weisen, die in ihrem Wissen den Göttern gleichen, zum anderen die vollkommen «Dummen, ohne Geist und ohne irgendwelche Fähigkeiten » (DA, II, 29o). Das Staunenkönnen leitet sich her vom Wissensstand, der Weisheit und der Intelligenz einer Person. Da die genannten Ausnahmezustände nur in der Literatur, nicht aber in der Realität vorkommen, können sie ausser Acht gelassen werden. Die Gruppe der Staunenden wird dreigeteilt:In die erste Gruppe gehören die Kinder, das Volk, die Frauen und die Ungelehrten, in die zweite Gruppe gehören die mittleren Gelehrten, zur dritten Gruppe werden die Gelehrten und die Philosophen gerechnet, die schon bei Aristoteles und Platon zu den Staunenden gezählt worden sind (DA, II, 292). Patrizi präzisiert diese Struktur der Rezipienten nicht in der Deca ammirabile, sondern in der Deca semisacra. Dort führt er aus, dass die Ungelehrten und die mittleren Gelehrten vor allem darüber staunen, was sie nicht kennen und wissen, während die Weisen über die ewigen und göttlichen Phänomene staunen. Daraus folgt, dass auch die Dichtung nicht alle Personen, sondern je nach Inhalt nur eine jeweilige Gruppe zum Staunen bringt: « Das heisst, dass nicht / jede Dichtung jeden Geist zum Erstaunen bringt » («Il perchè non / ogni poesia ogni ingegno farà maravigliare $[\ldots] », D S S$, III, 4O7). Die Weisen gilt es nicht (in erster Linie) mit Überraschungseffekten und Unerwartetem zum Staunen zu bringen, sondern mit einer Darstellungs- und Ausdrucksweise, die das Ewige erahnen lässt. ${ }^{116}$ Für den Dichter resultiert daraus aber nicht eine gezielte Ausrichtung seiner Tätigkeit auf ein spezifisches Rezipientenfeld, sondern die Pflicht, jeden Gegenstand wunderbar (mirabile) darzustellen, unabhängig davon, wer die Leser oder Zuhörer sein werden. Jedem Dichter ist von Natur aus die Begabung zu einem oder mehreren Genres gegeben und innerhalb dieser Genres soll er sich betätigen.

115 Der Euripos ist die schmalste Meerenge der Welt und zeichnet sich durch « ungewöhnliche Gezeitenströmungen » aus. Dieser wechselseitige, irreguläre und unberechenbare Charakter ist, so denke ich, auch ein Merkmal der potenza ammirativa. Vgl. https:// de.wikipedia.org/wiki/Euripos (Stand April 2021).

116 Gleichzeitig sagt Patrizi, dass Weise vielen Freuden verlustig gehen, da sie aufgrund ihrer Allwissenheit kaum mehr staunen können (DA, II, 367$)$. 
Da die maraviglia mit der erkennenden Potenz verbunden ist, kann sie nicht aus völliger Unwissenheit resultieren. Es bedarf einer gewissen, wenn auch noch unvollständigen Wissensbasis und eines Verlangens nach Wissen. Das Verlangen nach Wissen und die Freude am Lernen gehen mit dem Staunen einher. ${ }^{117}$ Patrizi zitiert Strabon, der das kindliche Streben nach Wissen mit der Freude an Märchen und Erzählungen verbindet:

«L'huom di natura è amico del sapere, e di ciò premio è l'essere egli amico alle favole, onde cominciano i fanciulli ad udire, e a partecipare di più lunghi ragionari. $\mathrm{E}$ di questo loro piacere cagione è che la favola è certa novità di parlare, che non dice le cose come elle stanno, ma oltre da questo, e dilettevole è ciò ch'è nuovo, e quello ch'altri prima non conobbe. E questo è appunto quello che ci fa amici / del sapere. Ma quando a ciò s'aggiunge il mirabile e il mostruoso più intenso si fa il diletto, il quale è dello imparare l'amoroso incanto. »118 (DA, II, 346-347)

In Erzählungen und Märchen wird Neues, bisher Unbekanntes erzählt, das zusätzlich intensiviert wird, wenn es mit Wunderbarem und Monströsem angereichert wird. Die fiktive Erzählung inszeniert ein gekonntes Spiel zwischen Glaub- und Unglaubwürdigem: Wir glauben Dinge, die wir wissen, gesehen, gehört oder gelesen haben, und wir sind ungläubig und staunen über Phänomene, die neu und unbekannt sind und unerwartet eintreten (DA, II, 365). Im Gegensatz zu den Aristotelikern, die das Staunen mit dem Glaubwürdigen zu verbinden suchen, koppelt Patrizi das Staunen an das Unglaubwürdige. Dieses ist - so Patrizi - das eigentliche Fundament des Wunderbaren und es wirkt umso stärker, als es an das Glaubwürdige gebunden bleibt und so die Distanz zu diesem noch stärker zu spüren ist ( $D A, \mathrm{II}, 310)$.

Adunque il mescolamento di ambedue, credibile ed incredibile, farà la maraviglia, ed il mirabile sarà non altro che un cotale congiungimento, di che di incredibili divengano credibili, o di credibili divengano incredibili. ${ }^{119}(D A$, II, 310)

117 Die Verbindung von Staunen und Lernen findet sich auch bei Aristoteles: Rhet., 1371b. Weitere Quellen sind im neunten Buch der Deca ammirabile zu finden.

118 Patrizi zitiert Strabon: Strabons Geographika, mit Übersetzung und Kommentar herausgegeben von Stefan Radt, Bd. 1: Prolegomena, Buch I-IV: Text und Übersetzung, Göttingen 2002, I, 2, 8: « Denn der Mensch hat Freude am Wissen, und die Vorstufe dazu ist die Freude an Fabeln: damit fangen daher die Kinder an, länger zuzuhören und an Gesprochenem teilzunehmen. Der Grund ist dass die Fabel etwas Neues erzählt: sie handelt nicht von dem Bestehenden sondern von Dingen die anders sind, und das Neue und bisher Unbekannte erfreut (eben das ist es auch was wissbegierig macht); wenn aber auch noch das Erstaunliche und das Wunderbare hinzukommt, erhöht das die Freude, die den Ansporn zum Lernen bildet.»

119 So wird die Mischung von beidem, Glaubwürdigem und Unglaubwürdigem, das Staunen ausmachen, und das Wunderbare wird nichts anderes sein als eine solche 
Die spannungsvolle Wechselwirkung zielt nicht darauf, aufgelöst zu werden, denn an das Staunen über das Unglaubwürdige sind Freude und Vergnügen gekoppelt. Im Vordergrund steht für Patrizi somit nicht, die Ursache von allem $\mathrm{zu}$ kennen und $\mathrm{zu}$ absolutem Wissen zu gelangen, sondern die Freude des Menschen am Neuen, am Lernen, am Staunen zu erhalten. Das Wissen, das mit Dichtung vermittelt werden kann, kann sowohl (natur-)wissenschaftlicher Natur sein, es kann aber auch ein prophetisches, enigmatisches, magisches Andeuten der höheren Wahrheit sein, das erstgenannte Wissensformen übersteigt. Patrizi fasst unter den Begriff der sapienza sowohl Wissen als auch Weisheit; der Klarheit halber versuche ich trotzdem, die Begriffe auseinanderzuhalten (z. B. DA, II, 239, 262).

Patrizi unterscheidet Phänomene, in denen das Staunen verblasst oder vom Wissen über die Ursache abgelöst wird, von Phänomenen, über die wir nie aufhören zu staunen, selbst wenn sie wissenschaftlich erklärt werden können wie der Himmel oder die Weltwunder (DA, II, 366$)$.

E ciò avviene nelle grandissime opere, non pur di Dio, ma degli huomini ancora, e per quanto dall'effetto del mirare le Piramidi di Egitto si pruova, anco gli altri sei miracoli del mondo è da credere che maraviglia continua di sè porgessero. ${ }^{120}$ $(D A$, II, 366$)$

Da diesen Werken eine göttliche Grösse und Schönheit inhärent ist, wird die maraviglia unaufhörlich befördert. Nebst dem göttlichen Charakter bestimmter Phänomene gibt es gezielte Mittel, die ein Ende des Staunens verhindern können. Paradigmatisch dafür steht das Enigma, das seit Anbeginn der Menschheit die Form göttlicher Botschaften und Prophezeiungen bestimmte und das aufgrund seiner Beschaffenheit - in seiner unauflösbaren Rätselhaftigkeit - das Staunen antreibt. Dies ist die von Patrizi bevorzugte Form des Staunens.

\subsubsection{Ausblick}

Obwohl Patrizi seine „psychologischen“ Erkenntnisse nur bedingt aus der empirischen Beobachtung des Menschen gewinnt, ist die Definition des Staunens als verbindende Bewegung zwischen Affekten und Kognition nicht nur herausragend für seine Zeit, sondern könnte auch in heutigen Diskussionen rund um die Dichotomie von Affekten und Kognition bzw. deren

Vereinigung, wenn aus Unglaubwürdigem Glaubwürdiges wird oder aus Glaubwürdigem Unglaubwürdiges entsteht.

120 Und das geschieht in den grösssten Werken, nicht nur von Gott, sondern auch von den Menschen, und so wie der Effekt des Staunens über die ägyptischen Pyramiden scheint sich auch bei den anderen sechs Weltwundern das Staunen immer weiter zu verbreiten. 
Überwindung eine zentrale Rolle spielen. ${ }^{121}$ In neueren Texten der Ästhetik und der Psychologie wird versucht, die Dualität zwischen Affekten und Kognition so aufzulösen, dass das eine in das andere integriert bzw. deren Interdependenz aufgezeigt wird. Nelson Goodman beispielsweise integriert sowohl die Sinneswahrnehmungen wie auch die Emotionen in den Bereich der kognitiven Kunsterfahrung:

Die meisten der Schwierigkeiten, mit denen wir uns herumgeschlagen haben, lassen sich [...] der dominierenden Dichotomie zwischen dem Kognitiven und dem Emotionalen zuschreiben. Auf die eine Seite stellen wir Sinnesempfindung, Wahrnehmung, Folgerung, Vermutung, jegliche nervenlose Untersuchung und Forschung, Tatsachen und Wahrheit; auf die andere Wohlgefallen, Schmerz, Interesse, Befriedigung, Enttäuschung, jegliche gehirnlose affektive Reaktion, Zuneigung und Abscheu. Dies versperrt, und das ziemlich wirkungsvoll, die Einsicht, dass Emotionen in der ästhetischen Erfahrung kognitiv funktionieren. Das Kunstwerk wird sowohl mit den Gefühlen, als auch mit den Sinnen erfasst. ${ }^{122}$

Eine andere Perspektive vertritt Luc Ciompi, der die Affekte als Motivatoren definiert und sie für die Tätigkeit des kognitiven Bereiches verantwortlich macht:

Affekte wirken als Motivatoren, sie bestimmen den Fokus der Aufmerksamkeit, sie öffnen „Schleusen [...] zu unterschiedlichen Gedächtnisspeichern“, schaffen Kontinuität, „bestimmen die Hierarchie unserer Denkinhalte“ und reduzieren Komplexität. ${ }^{123}$

Das Staunen spielt für diese Theorien kaum eine Rolle. Es wäre durchaus lohnenswert, analog zu Patrizis Bestimmung danach zu fragen, wo das Staunen in heutigen Theorien seinen Platz haben könnte.

Eine kommunikative Vermittlungsfunktion, wie Patrizi sie dem Staunen zuschreibt, wird in einigen Theorien der Imagination zugeschrieben. So hat schon Ficino das Staunen als Vermittler zwischen den Sinnen und der Vernunft, zwischen Körper und Geist begriffen. ${ }^{124}$ Dabei erfolgt eine klare Abwertung

121 Vgl. beispielsweise in den Neurowissenschaften Erich Schröger und Stefan KoE LSCH (Hgg.), Affektive und kognitive Neurowissenschaften, Göttingen 2013, S. 1-10.

122 Nelson Goodman, Sprachen der Kunst. Entwurf einer Symboltheorie, Frankfurt a. M. 1998 [1973], S. 228. Zitiert aus: Norbert R. Vetter, Emotion zwischen Affekt und Kognition. Zur emotionalen Dimension in der Kunstpädagogik, Köln 2010, S. 113.

123 Burkhard MeYer-Sickendiek, Affektpoetik. Eine Kulturgeschichte literarischer Emotionen, Würzburg 2005, S. 11. Meyer zitiert aus LuC CıомPI, Affektlogik. Über die Struktur der Psyche und ihre Entwicklung. Ein Beitrag zur Schizophrenie-Forschung, Stuttgart 1982, S. 97-98.

124 Desideri / Cantelli, Storia, S. 15 o. 
der Sinne gegenüber der Immaterialität der Vernunft. Die Seele insgesamt ist bei Ficino wie schon bei Platon immateriell gedacht, während Patrizi - wie selbstverständlich und unkommentiert - den körperlich-sinnlichen Teil miteinbezieht und zur Seele hinzurechnet. ${ }^{125}$ Im Gegensatz zu Platon und Ficino, die die Begierden abwerten und der Vernunft unterordnen, räumt Patrizi den körperlichen Begierden und dem Denken eine gleichberechtigte Position ein. Es scheint also, als würde Patrizi eine durchaus „moderne“ Position vertreten so dass auch hier ein Vergleich oder eine Analogie zu aktuellen Imaginationsund Kreativitätstheorien spannende Erkenntnisse bringen könnte.

\subsection{L'Eridano}

Patrizis enkomiastisches Poem L'Eridano ${ }^{126}$ lässt sich nur bedingt aus dem Blickwinkel der Poetica lesen, denn es erschien bereits 1557 - also knapp dreissig Jahre vor den ersten beiden Deche der Poetica. Es ist Kardinal Ippolito II. d'Este gewidmet und entwirft zum einen den Ursprungsmythos der stirpe estense, zum anderen prophezeit es die « sorti magnifiche degli eroi estensi ». ${ }^{127}$ In dem kurzen Poem ${ }^{128}$ findet sich eine Vielzahl der Themen und Kriterien, die Patrizi in der Poetica als Bestandteile seiner Poetik des mirabile und der maraviglia definieren wird. Allerdings handelt es sich in vielen Fällen um gängige Spezifika des Lobgedichts - dieses kann als prädestinierte Ausgangslage, nicht aber zwingend als Ausdruck einer Poetik des Staunens gelesen werden.

Im zweiten Vers kündigt Patrizi sein Poem als « mio nuovo altero canto » an, als Gesang also, der Neues und Würdevolles verkünden wird. An diese Worte

125 Patrizi folgt in seiner Konzeption der Seele mehrheitlich dem aristotelischen Verständnis der anima, das über Avicenna in der Renaissance Verbreitung fand. Darin werden folgende mentale Bereiche unterschieden: der sensus communis (wo alle Sinneseindrücke zusammenfliessen) und die imaginatio als ein erster Bereich, die intellektuellen Fakultäten (Denken, Vernunft, Verstehen) in einem zweiten Bereich und die Erinnerung in einem dritten Bereich. Siehe Martin Ke mp, From „Mimesis“to „Fantasia“: The Quattrocento Vocabulary of Creation, Inspiration and Genius in the Visual Arts, in: Viator 8 (1977), S. 362 .

126 Der „Eridanus“ ist ein Fluss, der in der ägyptischen Mythologie als Himmelsfluss, in der griechischen Mythologie als Fluss am Ende der Welt, entstanden aus Okeanos und Thetys, bezeichnet wird. Verschiedentlich wurde der Eridanus mit der Rhone oder dem Po in Verbindung gebracht; « Herodot und Strabon hielten den Eridanos für rein mythisch [, b]ei Vergil erscheint der Eridanus als Fluss der Unterwelt ». Siehe https://de.wikipedia. org/wiki/Eridanus_(Mythologie) (Stand April 2021).

127 Borsetto, Utopia, S. 185.

128 L'Eridano setzt sich aus 650 Versen mit dreizehn Silben zusammen. 
ist ein Selbstverständnis des Dichters geknüpft, der nicht auf bereits Bekanntes zurückgreift, sondern in seinem Gedicht die (fiktiven) Ursprünge und die (prophetische) Zukunft neu und frei kreiert. Ergänzend bittet er Apollon, ihm Furor einzuflössen, damit er innerlich vom « ewigen Ruhm der unsterblichen estensischen Helden » erfüllt und zum Schreiben angeregt werde (Vv. 9-11).

Der Mythos rund um die estensische Adelsfamilie beginnt mit Vers 12. Erzählt wird von der Nymphe Ferrara, Tochter des Po, die ein schlimmes Schicksal erleidet: Ihre Ehemänner sterben einer nach dem anderen und lassen sie als trauernde, einsame Witwe zurück. Verzweifelt bittet sie ihren göttlichen Vater Po um Hilfe, er solle entweder ihr Schicksal in ein freudiges oder aber sie selbst in eine andere Gestalt verwandeln (Vv. 31-46). Po staunt über ihre Worte und verspricht ihr, die « tormenti » in « letizia » zu verkehren:

Et ella pur tutt'or piangendo, con parole D'alti singulti e da sospir ardenti rotte, Al padre venne ad una ad una sue sciagure Dal dì che nacque in fasce in fin alor contando.

Le quali 'l Po sentendo sopra sua credenza Gravose assai, di stupor pieno e di pietade Teneramente pianse. E vòlto a la figliuola - Pon omai - disse - giù l'alta amarezza e i pianti, Mia figlia: per che fin agli aspri tuoi tormenti Tosto vedrai, e 'n altretanta tua letizia Conversi. E Dio di tanto t'assicura e padre. $-(\mathrm{Vv} \cdot 70-80)^{129}$

Patrizis Sprache zeichnet sich durch eine Vielzahl von Alliterationen zum Beispiel jene auf "p“, die den „padre“, „Po“ und die „pianti“ der Tochter aneinanderbinden - und Enjambements aus, die das Fliessen der Verse gleichzeitig garantieren und brechen. Der zugleich natürliche und künstliche Fluss der Verse spiegelt sich in der Figur des Po, der sowohl in seiner Naturgestalt als auch als literarische Figur Eingang in den Text findet.

Das Staunen des Po («'l Po [...] di stupor pieno ») überträgt sich auf die Wellen des Flusses, denn diese kommen durch das intensive Nachdenken und

129 Ich zitiere aus der bereits genannten Ausgabe von CARDUCCI, La poesia barbara. Die Übersetzungen stammen von der Autorin (A.E.): « Und sie, auch wenn rund um die Uhr weinend, kam mit Worten, / die von hohen Schluchzern und glühenden Seufzern unterbrochen wurden, / zum Vater, um von jedem einzelnen Unglück / vom Tag, als sie geboren wurde, bis zum heutigen Tag zu erzählen. / Als Po diese hörte, viel schwerwiegender als er / geglaubt hatte, weinte er zärtlich voller Staunen / und Mitleid. Und der Tochter zugewandt: / - Lege ab - sagte er - die grosse Bitterkeit und die Tränen, / meine Tochter: Denn am Ende deiner herben Qualen / wirst du bald sehen, wie diese in ebenso grosse Fröhlichkeit / umgewandelt wird. Das versichere ich dir als Gott und Vater. » 
die Trauer ihres Herrn zum Stillstand. Damit gleichen die Klagen des Po jenen des Orpheus:

[...] E vide alor il pescator spantato

Fermato 'n sé 'l gran fiume, e l'onde alte et immense,

Perdute in quel dolor, istar stupide e immote. ${ }^{130}$ (Vv. 87-89)

Der Stillstand der Wellen wird durch den Verzicht auf Enjambements bekräftigt, gleichzeitig wird damit die Unterbrechung der Handlung durch das Innehalten des Flussgottes markiert.

Wenngleich Pos Reflexion in ihrer Wirkung dem Gesang des Orpheus ähnlich ist, ist es nicht der göttliche Dichtersänger, der Po als Ausweg für seine Tochter erscheint - schliesslich vermochte dieser sein eigenes Schicksal auch nicht zu wenden -, sondern der grosse Meister der Verwandlung: Proteus. In der Figur des Proteus, polymorpher Meeresgott mit prophetischer Gabe, vereinen sich die beiden Hauptmotive des Gedichts: die Prophezeiung der gloriosen Zukunft der Este und die Verwandlung von Ferrara in den mythischen Ursprung der Adelsfamilie. ${ }^{131}$ Im Gegensatz zu Platon, der die Mythen rund um Proteus als Lügengeschichten deklassiert, ${ }^{132}$ stellt Patrizi dessen Metamorphosen ins Zentrum seines Gedichts. Auch in der Poetica wird er die Verwandlung als wirkungsstarkes Mittel preisen. So lobt Patrizi beispielsweise die Bombyces von Vida aufgrund der in ihnen dargestellten Verwandlungen:

Ma la Bombice sua e da divinità è derivata e da natura, e d'arte umana canta, e nel vero il soggetto per sua natura è maraviglioso per le tante trasformazioni che [ha] di sè il verme della seta, ed è assai pienamente ornata di trova/ti propri al poeta, e di favole, e di ornamenti di dir bellissimi, che a pieno la fanno poetica e mirabile d'ogni parte $[\ldots]^{133}(D S S$, III, 418)

$130[\ldots]$ Und es sah also der ruinierte Fischer / in sich gekehrt den grossen Fluss, und die hohen und immensen Wogen, / verloren in diesem Schmerz, blieben erstaunt und unbeweglich.

131 Siehe auch die Verse 93-94. Patrizi erweitert damit die Sage rund um Proteus, die schon von Ariosto im Orlando Furioso (Canti VIII und XI) auf originelle Weise in das fantasievolle Geschehen des romanzo eingebunden wurde. Ariosto erzählt von der Insel Ebuda, deren Bevölkerung als Rache dem Proteus - der die Tochter des Königs liebte und schwängerte, woraufhin diese von ihrem Vater getötet wurde - jeden Tag eine Jungfrau opfern und die Insel vor der Meeresherde (VIII, 54, V. 6) beschützen muss. Schliesslich wird Orlando die Insel von ihrem bösen Fluch befreien (XI).

132 Polit., 381d: «Und es soll uns auch keiner Lügengeschichten von Proteus und von Thetis erzählen, und weder in Tragödien noch in anderen Gedichten lasse einer die Hera in der Gestalt einer Priesterin auf die Bühne treten [...] »

133 Aber die Bombyces leiten sich vom Göttlichen und von der Natur ab und besingen die menschliche Kunst, und in Wahrheit ist der Stoff natürlicherweise erstaunlich aufgrund 
Die Verwandlungen der Seidenraupe, die eigenen Erfindungen des Dichters, die Erzählung und die sprachliche Ausgestaltung verleihen dem Werk Vidas seinen poetischen und erstaunlichen Status. Genauso wie Vida wählt Patrizi mit Ferrara und dem Po zwei Subjekte, die ursprünglich der Natur bzw. der Landschaft entstammen, vom Dichter aber mythisch angereichert und überhöht werden. Patrizi setzt wie Vida seine poetische Kreation dazu ein, eine Stadt an den Ufern des Po - Cremona bei Vida, Ferrara bei Patrizi - mit einem neuen Ursprungsmythos zu unterlegen. Beide Autoren wählen einen Verwandlungskünstler als zentrale Figur ihrer Dichtung.

Da Proteus seine Herde in den griechischen Gewässern nur ungern verlässt, ist ein Wunder nötig, um ihn an die Ufer des Po zu locken (V. 144). In einem tosenden Gewitter verliert der Meeresgott seine Herde und findet sie nach langer Suche an den Ufern des Po wieder (Vv. 200-205). Die Söhne des Po fesseln den schlafenden Gott, und als dieser aufwacht, verwundert um sich blickt («mira intorno») und erkennt, wie ihm geschah, verwandelt er sich erfolglos in verschiedene Gestalten, um den Fesseln zu entkommen. Die göttlichen Kräfte sind wirkungslos in dieser neuen Variante des Mythos, also bittet Proteus Po um Hilfe. Dieser verspricht die Freilassung unter der Bedingung, dass er das Schicksal seiner Tochter zum Guten wende. Daraufhin sagt Proteus Ferrara einen Ehemann voraus, der von Herkules abstamme und mit dem sie das ruhmreiche, ewige Geschlecht der Este begründen werde. Der schwarze Adler des Jupiter werde sich in einen weissen verwandeln - das Wappen der Este. Proteus schliesst mit der Prophezeiung eines neuen und jungen Sängers, der die Erinnerung der Este hochhalten wird - dieser Sänger wird, wie sich später im Gedicht herausstellt, Patrizi sein:

E questo fia quando uno di nazïon straniera

Giovane assai in su le rive tue ferrigne

Udrai temprar altera nuova cetra etrusca.

Allor fa che le Muse e il divo Apol convite

Et oda il nuovo altiero suon, ché questo è il tempo

Dal ciel prescritto, che, viventi i tuoi nipoti,

Là suso in te le nove stelle più lucenti

Splendan fiammanti, a Giove lor avolo care. - ${ }^{134}$ (Vv. 297-299)

der vielen Verwandlungen, die die Seidenraupe durchmacht, und ist reich geschmückt mit Erfindungen des Dichters und Erzählungen und wunderschönem Wortschmuck, die sie gänzlich poetisch und wunderbar in allen ihren Teilen machen.

134 Und das war, als du einen ziemlich jungen Mann / von fremdländischer Herkunft an deinen eisernen Ufern / mit der stolzen, neuen, etruskischen Zither spielen hörtest. / Führe die Musen und den heiligen Apollon zusammen / und höre den neuen erhabenen Gesang, denn dies ist die Zeit, / die vom Himmel vorgeschrieben ist, und zur Zeit deiner 
Die «altera nuova cetra» wie auch der «nuovo altiero suon» wiederholen wörtlich den Beginn des Poems und werden auch gegen Ende («nuovo canto », V. 543) noch einmal aufgegriffen. Das Neue wird zum kennzeichnenden Merkmal der Dichtung, wobei es sich vor allem in der Neuartigkeit des Mythos offenbart: Ist die Verwandlung normalerweise Proteus' Mittel, um den Prophezeiungen, die von ihm eingefordert werden, zu entkommen, ist es im Eridano gerade die Prophezeiung, die ihn erlöst. Die Verwandlung wird vom Mittel des Gottes zum Mittel des Autors, wenn dieser das Schicksal der historischen und mythischen Figuren nach seinem Gutdünken verändert.

Entsprechend den Worten des Proteus vermählt sich Ferrara mit Azzo, zudem wird ein Gastmahl veranstaltet, an dem die benachbarten Nymphen und zufliessenden Flüsse (V. 445), die griechischen Götter und Flüsse, Ippolito mit seinen Geschwistern und Kindern sowie die Königin Renata anwesend sind. Mythisches und historisches Personal treten gemeinsam auf und gehen zum Palast der Este, über eine Strasse, die Po « übernatürlicherweise mit erstaunlichem Vorgehen / in seinen Gewässern geformt hat» («oltra natura con mirabil modi / Fatta avea 'n l'acque sue», Vv. 493-494) - ein Wunder, das in die Erzählung eingebettet ist.

In der Poetica wird Patrizi Vida dafür loben, als Setting für sein Poem Scacchia Ludus einen «convito » der Götter gewählt zu haben und damit das Menschliche hat göttlich werden lassen.

Ma la Scacchia del Vida, che pure arte tratta ed è per sè umile assai, con maravigliosa maniera è spiegata, pienissima / di bellezza della favella e poetica oltre a modo. Ma l'invenzione del convito fatto a' dei in Etiopia, e dopo il convito per trattenimento de' convitati, fatti portare gli scacchi, il farvi giocare Mercurio e Apollo, con certe leggi, fu dilettevolissima finzione, con la quale quello che era umano fa essere divino, molto più altamente che non fece Omero nella guerra $[\ldots]^{135}(D S S, 418-419)$

Analog erhebt Patrizi im Eridano nicht nur die Este, sondern auch sich selbst auf eine Ebene mit den Göttern. Denn nachdem die Menge im Palast feierlich

Enkel / werden da unten die hellsten und jüngsten Sterne in dir / Erstrahlen, ihrem Grossvater Jupiter teuer.

135 Aber die Scacchia von Vida, auch wenn sie von einer Kunst handelt und an sich ziemlich bescheiden ist, wird auf erstaunliche Weise erklärt, voller / erzählerischer Schönheit und überaus dichterisch. Aber die Erfindung des Gastmahls zu Ehren der Götter in Äthiopien, und dass diese nach dem Mahl zur Unterhaltung zum Schachspiel geführt wurden, und er Merkur und Apollon hat spielen lassen, nach bestimmten Regeln, war eine überaus vergnügliche Erfindung, in der das, was menschlich war, göttlich wurde, noch viel grösser als Homer dies im Krieg hat geschehen lassen [...] 
gespeist hat, fängt Apollon an zu singen - begleitet von Patrizis Zeitgenossen Gabriele Cesano (V. 516), Vicenzo Maggio (V. 520), Giacopo Canano (V. 522), Giraldo Cinzio (V. 523), Giovan Battista Pigna (V. 525) und weiteren -, bevor er die «divina cetra » in die Hände Patrizis übergibt. Wie die Anfangszeilen des Gedichts wird die folgende Stelle in der Ich-Perspektive wiedergegeben:

E poi ch'ei tacque a me si fece, e lieto in mano

Mi pose la divina cetra, e sorridendo

Baciommi in bocca: - Or a te tocca - disse -, e alterno

Risponderan le Muse e de' tuo' amici 'l coro. -

Pien dunque io di calor e di furor divino,

Danzando in giro il coro e stando io fermo in mezo,

Cominciai nuovo canto; e rispondeami 'l coro.

Patricio. Càngiati, Apollo; e questi altier celesti eroi

Ne l' Eridan instella: ormai càngiati, Apollo.

Coro. Càngiati Apollo; e questi altier celesti eroi

Ne l'Eridan instella: ormai càngiati, Apollo. ${ }^{136}$ (Vv. 537-547)

Patrizi bittet Apollon in seinem Gesang darum, sich zu verwandeln und die estensischen Heroen als Sterne des Eridanus aufleben zu lassen. „Instellare“ ist ein seltenes Wort und damit Teil der «lingua straniera», die Patrizi in seiner Poetica einfordern wird (DA, II, 255) ${ }^{137}$ Ich hebe es hervor, weil es im zitierten Gesang des Patrizi und in den Passagen des Chors bis zum Ende des Gedichts weitere neun Male wiederholt, bekräftigt und in die memoria der Leser eingeschrieben wird.

Die finalen Passagen des Dichtersängers Patricio sind als Lob und Bewunderung der estensischen Machthaber auf der einen, ${ }^{138}$ als Bitte um deren Schutz und Kraft auf der anderen Seite konzipiert. Patrizi wiederholt gewissermassen die von Proteus dargebrachte Prophezeiung und bekräftigt sie durch die Wiederholung in der Form eines rituellen Gesangs. Angelangt

${ }_{13} 6$ Und nachdem er geschwiegen hatte wandte er sich mir zu, und mit leichter Hand / reichte er mir die göttliche Zither, und lachend / küsste er mich auf den Mund: - Jetzt ist es an dir - sagte er - und alternierend / werden die Musen und deine Freunde dir im Chor antworten. - / Ich war also voller Hitze und göttlichem Furor, / der Chor umhertanzend und ich mittendrin stehend, / und begann einen neuen Gesang; und es antwortete mir der Chor / Patricio. Verwandle dich, Apollon; und mache diese stolzen himmlischen Helden / im Eridano zu Sternen; verwandle dich, Apollon. / Chor. Verwandle dich, Apollon; und mache diese stolzen himmlischen Helden / im Eridano zu Sternen; verwandle dich, Apollon.

137 Siehe dazu das Lemma « instellare » im Vocabolario degli Accademici della Crusca: $\mathrm{http}: / /$ www.lessicografia.it $/$ pagina.jsp?ediz $=5 \&$ vol $=8 \&$ pag=951\&tipo=3 (Stand April 2021).

138 Ercole II. wird von der « betrübten, nachlässigen, alten und / bettelnden Italia » (Vv. 577578), Alfonso II. vom Kriegsgott Mars (Vv. 612-613) bestaunt. 
bei seinen Zeitgenossen - Ercole II., Luigi und Alfonso II. - richtet der Sänger einige Verse an sich selbst: Er bezeichnet sich als «forte e franco / Guerrier », als Erneuerer der antiken Werte:
$P$. E tu, o Francesco, più d'ogn'altro forte e franco
Guerrier, che sol l'ardir et il valor antico
De l'alme italich' a dí nostri rinovelle:139 (Vv. 589-591)

Mit einem humoristischen Unterton fügt er an, die „alma italica“ könne ihm zwar keine Herrscherkrone aufsetzen, doch als Trost sei ihm ein Stern des Eridanus beschieden und werde seine Stimme von den «starken Rittern» herbeigebeten (Vv. 595-598). Wie schon in Polizianos Ambra preist der Dichter sich als wagemutigen Helden, dem dieselbe Bewunderung wie dem politischen und kriegerischen Helden entgegengebracht wird und der dieselbe hohe gesellschaftliche Stellung geniesst. Auch Ruhm und Ehre werden dem Dichter gleichermassen zuteil, wird er doch in einen ewig leuchtenden Stern verwandelt.

Zum Schluss wird der Gesang Patrizis und seines Chors erhört: Apollon verwandelt sich in einen weissen Adler, « und die Namen dieser Helden, die in seine Krallen / eingeschrieben waren, erhob er zum Himmel» (V. 646). Durch den rituellen Gesang wird die mythische Verwandlung, die von Proteus prophezeit wurde, in Gang gesetzt - Gott und Dichter verschränken sich in ihren Rollen als Propheten und Verwandlungskünstler.

Die Qualitäten des mirabile und der maraviglia, wie Patrizi sie in der Deca ammirabile theoretisch aufarbeiten und definieren wird, können bereits im frühen Poem eruiert werden - wenngleich sie noch keine systematische Poetik der maraviglia definieren: ${ }^{140}$ Der Enthusiasmus sowie der göttliche Furor werden zu Beginn des Textes wie auch vor dem Schlussgesang an Apollon genannt. Der Gesang offenbart eine göttliche Prophezeiung und birgt in dieser wie auch im Motiv der Verwandlung eine enigmatische Komponente. Insbesondere zeugt die Übertragung der metamorphotischen Fähigkeiten vom Meeresgott auf den Dichter von den ausserordentlichen, ja magischen Fähigkeiten des Letzteren. Als Charakteristika der Erzählung wird Patrizi in der Poetica das Neue, Fiktive und Ausserordentliche definieren, zudem wird durch den Verweis auf den philomythos in Aristoteles' Metaphysik die mythische Komponente des Erzählten betont (DA, II, 264). Alle vier Eigenschaften der

$139 P$. Und du, oh Francesco, stärker und ehrlicher als jeder andere / Krieger, der als einziger die Kühnheit und den antiken Wert / der italischen Seelen bis in unsere Tage erneuerst:

140 Entusiasmo, profezia, enimma, sapienza, favola, allegoria, varietà, aggrandimento, sminuzzamento, evidenza, dolcezza, lingua straniera, verso, canto. 
Erzählung sind im Eridano umgesetzt: Das Poem weist eine neue, nie gehörte favola auf, die vom Autor erfunden wurde und Ausserordentliches über den griechischen Gott Proteus wie auch über die Este erzählt. Teil der favola ist die Allegorie, wie wir sie im Falle der Personifizierung von Po und Ferrara antreffen. Sie dient der Mythisierung wie auch der Veranschaulichung des Erzählten, indem sie Orten und historischen Figuren ein Gesicht und eine Geschichte verleiht.

Die Vielseitigkeit (varietà) des Gedichts ist gegeben durch die Einbindung historischer wie auch mythischer und fiktiver Elemente, ebenso wird die Sprache an den erzählten Inhalt angepasst. Ein aggrandimento des erzählten Inhalts finden wir bezogen auf die Este auf der einen, auf den Autor auf der anderen Seite. Beide werden Teil der griechischen Götter- und Heldentradition, beiden wird eine glorreiche Zukunft vorausgesagt. Auch die sprachlichen Eigenschaften, wie sie in der Poetica bestimmt werden, finden sich im Eridano vereint: Dolcezza zeigt sich in der fliessenden Versbildung, lingua straniera finden wir in zentralen Begriffen wie „instellare“ sowie im ungewöhnlich betonten Vers mit dreizehn Silben:

[...] il Patrizi costruisce un verso di tredici sillabe sciolto da rima con accenti principali di quarta, di ottava e di dodicesima sul modello dell'endecasillabo trissiniano, ma diverso da questo per ampiezza di respiro musicale e «straordinarietà » di effetto ritmico. ${ }^{141}$

Der Gesang (canto) ist als Gesamtstruktur des Textes, insbesondere aber im Gesang des Patricio im Wechsel mit dem Chor am Ende des Poems umgesetzt. Sind die zentralen Qualitäten des erstaunlichen Gesangs vorhanden und teilweise explizit genannt - insbesondere das Prophetische und das Neue -, wird die wirkungsästhetische Komponente der maraviglia im Poem kaum angesprochen. ${ }^{142}$ Ruhm und Ehre sind die erwünschten Folgen des dichterischen Gesangs, Bewunderung und Staunen können höchstens implizit als Reaktionen auf den verwandelten Mythos mitgedacht werden. Es scheint naheliegend, dass Patrizi die Wirkung der maraviglia als Pendant zum mirabile erst in späteren Jahren konzipiert hat. Die Übereinstimmung der literarischen Qualitäten im Eridano und in der Poetica deutet allerdings darauf hin, dass das Lobgedicht - das unter anderem mit der Hymne verwandt ist - als Genre eine Vielzahl von Qualitäten liefert, die in Patrizis Poetik des Staunens eingeflossen sind.

141 Borsetto, Utopia, S. 206.

142 Einzige Ausnahme ist Pos Staunen über Ferraras Bericht ihres traurigen Schicksals (V. 75). 


\subsection{Exkurs: Patrizi und der Manierismus}

Matuschek sieht Patrizis Poetica als paradigmatisches Beispiel für die Überführung der «Poetik vom Cinque- zum Seicento», die er als Ablösung der aristotelisch orientierten Poetiken durch die manieristischen beschreibt. Der Manierismus gipfle darin, dass er sich ganz dem movere hingebe und prodesse und delectare hinter sich lasse: «Dichtung [...] als verblüffende Wortartistik, das Staunen, das sie erregt, [...] als Maß ihres Gelingens. $»^{143}$

Die Diskussion um den Manierismus und dessen Bedeutung wird in der Literatur- wie auch in der Kunstgeschichte sehr ausführlich und umstritten geführt $^{144}$ - insbesondere die Definition des Begriffs ist vielfältig und variiert je nach Perspektive.

Eine wertneutrale Definition bietet Ernst Robert Curtius in seinem Versuch, den Begriff für die Literaturwissenschaft fruchtbar zu machen. ${ }^{145}$ Er beschreibt den Manierismus als Komplementärerscheinung zu klassischen Epochen, als Strömung, die nicht auf nachahmbaren, lehrbaren Prinzipien beruht und nicht die Angemessenheit am Gegenstand zum Ziel hat. Laut dieser Definition werden sowohl Form als auch Inhalt derart zugespitzt, dass Überraschendes und Scharfsinniges (acutezza) zum Ausdruck kommt. Von den Stilmitteln, die Curtius als spezifisch manieristische anfügt - dem Hyperbaton, der Periphrase, der Rätselrede, der annominatio und der marinierten Metaphorik -, findet sich in der Poetik Patrizis einzig die Rätselrede bzw. das Enigma.

Es ist richtig, dass Patrizi eine bewusste Differenz der dichterischen zur Alltagssprache proklamiert - das ist an sich aber noch keine antiklassische Tendenz, findet sich diese Forderung doch bereits in der antiken Rhetorik. ${ }^{146}$ Für Patrizi manifestiert sich das Aussergewöhnliche der poetischen Sprache in der Verwendung des Verses, der aber weiterhin den Prinzipien der Harmonie folgen soll, sowie der lingua straniera. Die lingua straniera zeichnet sich durch ihren inhaltlich verdichteten, ja prophetischen Gehalt aus. In seiner früheren Schrift zur Rhetorik (Della retorica, 1562) prangert Patrizi eine Sprache, deren Gehalt sich hinter einem eleganten Ornamentum verbirgt, explizit an. Obwohl diese Aussage auf die Rhetorik bezogen ist - deren Reduktion der Sprache

\footnotetext{
143 Matusche K, Über das Staunen, S. 136.

144 Eine gute Übersicht findet sich in Horst Brede kamp, Manierismus. Zur Problematik einer kunsthistorischen Erfindung, in: Wolfgang Braungart (Hg.), Manier und Manierismus, Tübingen 2000, S. 109-130.

145 Ernst Robert Curtius, Europäische Literatur und lateinisches Mittelalter, Bern / München 1967 [1948], S. 277.

146 Rhet., 1404b. Aristoteles konnotiert die Abweichung von der gewöhnlichen Alltagssprache mit Bewunderung und Staunen.
} 
zu einem Machtinstrument er ebenfalls stark kritisiert -, dürfte dies durchaus auch für die poetische Sprache gelten. ${ }^{147}$ Eine « ,Hypertrophie der Kunstmittel [ [... auf Kosten des Gehalts », wie sie «zum festen Bestandteil [...] der Definition des Manierismus » gehört, ist für Patrizi damit sicherlich nicht zutreffend. ${ }^{148}$

Patrizi sucht eine Sprache, die angelehnt ist an die Orakelsprache der Götter, die Weisheit beinhaltet und einen gemeinschaftsstiftenden Charakter besitzt, die also durchaus mit sinnvollem Inhalt gefüllt ist. In der Deca sacra wie auch in der Deca semisacra, die sich den verschiedenen Stoffen und deren sprachlichen Ausgestaltungen zuwenden, fällt auf, dass alle besprochenen Stoffe eine gesellschaftliche Relevanz aufweisen, seien es religiös-rituelle Texte oder aber Lehrtexte - zum Beispiel über die Künste des Menschen, über Moral und Geschichte. Der Inhalt ist also keineswegs beliebig, sondern erfüllt eine gesellschaftliche Funktion. Das Staunen ist zwar das übergeordnete Ziel, auf dieses folgen aber unmittelbar zuerst das Ziel des Lernens und als drittes Zieldas Vergnügen (DS, III, 414).

Patrizis Poetik entspricht teilweise den Prinzipien des Antiklassischen, dem «Widerstand gegen Prinzipien des Maßes, der Ordnung und der Regel » sowie dem paradoxalen und widersprüchlichen Charakter (z. B. credibile vs. incredibile), wie Arnold Hauser ihn für seinen Manierismusbegriff definiert und wie er ihn unter anderem bei Tasso und Marino am Werk sieht.

Erst aus der Spannung zwischen Klassik und Antiklassik, Naturalismus und Formalismus, Rationalismus und Irrationalismus, Sensualismus und Spiritualismus, Traditionalismus und Neuerungssucht, Konventionalismus und Revolte gegen jeden Konformismus lässt sich ein brauchbarer Begriff des Manierismus gewinnen. ${ }^{149}$

Hauser weist richtigerweise darauf hin, dass der Begriff des Antiklassischen wie Walter Friedländer ihn geprägt hat ${ }^{150}$ - nicht ausreicht und der Ergänzung durch die oben genannte Spannung bedarf. Diese paradoxale Spannung findet sich auch bei Patrizi, der in Anlehnung an Longin das Paradoxe als Quelle des Staunens miteinbezieht. Allerdings stiessen weitere Konnotationen, wie Hauser sie beschreibt und wie sie auch von der Tasso-Forschung als «manieristische Geisteshaltung » gefasst worden sind - Krise, Entfremdung,

\footnotetext{
147 Vasoli, Francesco Patrizi da Cherso, S. 91.

148 REgN, Tasso, S. 114.

149 ARnold HaUSer, Der Ursprung der modernen Kunst und Literatur. Zur Entwicklung des Manierismus seit der Krise der Renaissance, München 1964, S. 12.

15 O Walter Friedländer, Die Entstehung des antiklassischen Stiles in der italienischen Malerei um 1520, in: Repertorium der Kunstwissenschaft, Bd. 46 (1925), S. 49-86.
} 
innere Unruhe und Unsicherheit -, bei Patrizi kaum auf fruchtbaren Boden. ${ }^{151}$ Es wäre sowieso zu fragen, ob diese nicht eher für die Moderne, als deren Wegbereiter der Manierismus konzipiert wird, denn für die Renaissance passend sind. Patrizis Poetik zeugt nicht von einer « crisi del rinascimento », viel eher sieht er in einer schöpferischen Literatur, wie sie im frühesten Griechenland praktiziert wurde, einen vielversprechenden Zufluchtsort. Auch die Auseinandersetzung mit Aristoteles ist kein Zeugnis einer Krise, sondern Darlegung einer alternativen Sicht auf die Funktion, die Form und den Gehalt der Dichtung.

\subsection{Konklusion}

Um Patrizis Konzeption des Staunens fassen zu können, gilt es nicht nur, die Bewegung des Staunens zwischen potenza affettuosa und potenza conoscitiva nachzuzeichnen, sondern auch, die Bewegung seines Denkens zwischen historischer Quellenaufarbeitung, syllogistischem Be- oder Widerlegen und spekulativen, mythisch begründeten Bestimmungen zu begreifen. In der Deca ammirabile wird der systemische Kern von Patrizis Poetik dargelegt. Auf diesen laufen die historischen Aufarbeitungen der Deca istoriale und der Deca disputata zu, aus ihm gehen die nachfolgenden Deche hervor bzw. an ihn werden diese rückgebunden. Die Quellen signalisieren auf der einen Seite eine enzyklopädische Aufarbeitung, auf der anderen Seite zeichnet Patrizi mit ihnen seinen eigenen Denk- und Argumentationsprozess nach und lässt den Leser an diesem teilhaben. Die beiden Kernelemente der Dichtung - das mirabile und die maraviglia - werden in einem syllogistischen Verfahren als übergeordnete poetologische Kriterien herausgeschält, wobei sich Patrizis Logik vorwiegend an der Geschichte und an empirischen Erfahrungswerten, nicht an einer vorgegebenen theoretischen Basis orientiert.

151 Regn, Tasso, S. 108. Der «Problematik des modernen Menschen» hat sich auch der Curtius-Schüler Gustav René Hocke in seinen Manierismus-Studien vertieft angenommen. Er fokussiert auf die « Elemente[...] einer Tiefen-Ästhetik », die eine « irreguläre Ästhetik des Problematischen» ist und die menschliche Suche nach der «Urwahrheit» und dem «Absoluten» widerspiegelt. Hocke forscht vornehmlich zum Irrationalen und Irregulären, zum Esoterischen und Hermetischen - zur labyrinthischen Tiefenstruktur, wie er sie nennt -, während Patrizis Vorgehen, insbesondere auch seine Suche nach den (literarischen) Ursprüngen, klassisch-systematisch bleibt. Siehe Gustav René Hocke, Manierismus in der Literatur. Sprach-Alchimie und esoterische Kombinationskunst, Reinbek bei Hamburg 1959; ebenso De RS., Die Welt als Labyrinth. Manierismus in der europäischen Kunst und Literatur, Reinbek bei Hamburg 1987. 
Patrizi sieht in der Dichtung die Möglichkeit, die Vergangenheit heranzuholen, die Gegenwart zu begreifen und die möglichen Formen der Zukunft zu erahnen. ${ }^{152}$

Ähnlich umfassend wird der Rezipient konzipiert, denn durch die potenza ammirativa werden alle Elemente seiner Seele, seines Wahrnehmens, Fühlens und Erkennens einbezogen. Werden in der aristotelischen Metaphysik nur der Geist, in der Rhetorik des Aristoteles nur die Affekte thematisiert, fügt Patrizi beide Teile in seiner Poetica zusammen.

L'elemento essenziale della poesia che il Patrizi ha individuato - il mirabile, forma della poesia, la meraviglia, suo unico fine - coinvolge dunque tutto il testo poetico, non solo nella totalità dei suoi livelli, ma anche nei suoi rapporti col lettore. ${ }^{153}$

Die Verbindung von Wunderbarem und Staunen, wie Aristoteles sie im Mythos angelegt sieht, integriert Patrizi in sein Verständnis der Dichtung und macht sie zum Zentrum seiner Poetik. Das mirabile und die maraviglia sind die einzigen universell gültigen Qualitäten der Dichtung, sie setzen sich aus den Eigenschaften des Dichters, des poetischen Textes sowie des Rezipienten zusammen. Letztere ist ganz besonders zentral, denn ohne die Wirkung des Staunens kann auch keine Definition des mirabile im Text stattfinden - die maraviglia ist notwendige Eigenschaft, während die einzelnen Eigenschaften des Textes allein oder zusammen als hinreichende Eigenschaften betrachtet werden können. ${ }^{154}$

Patrizi setzt die Poetik der admiratio, wie Pontano sie konzipiert hat, in italienischer Sprache fort. Während Pontano sich vorwiegend an der Aeneis orientierte, vervielfältigt Patrizi seine Bezugspunkte und bezieht literarische, vorwiegend aber rhetorische und poetologische Texte von antiken und zeitgenössischen Autoren bei. Dabei öffnet er den poetologischen Blick in die früheste Vergangenheit, indem er sich auf älteste Textzeugnisse beruft, wie auch in die Zukunft, für die er mitunter die Möglichkeit neuer Textformen und Genres - als Kombinatorik der dem mirabile zugrundeliegenden Eigenschaften - offenhält. Patrizis Poetik ist in ihrer Anlage sicherlich der bisher wissenschaftlichste und umfangreichste Text; die darin

\footnotetext{
$15^{2}$ Bolzoni, L'universo, S. 111.

153 Ebd., S. 140.

154 Hennig befasst sich ausführlich mit der „kombinatorischen“ Methode, die Patrizi für die Gestaltung des mirabile vorlegt. Allerdings verkennt sie, dass nicht die kombinatorische Zusammensetzung das Mass des mirabile bestimmt, sondern die maraviglia aufseiten des Rezipienten. Damit wird die mathematische Berechenbarkeit unmittelbar unterlaufen. Siehe Hennig, Francesco Patrizi, S. 133.
} 
enthaltene Quellenanalyse beansprucht Vollständigkeit, die daraus abgeleitete Argumentation logische Nachvollziehbarkeit.

Wie Pontano hebt Patrizi das Versmass und die Fiktion als die zentralen Mittel der Dichtung hervor, zudem sieht er im mirabile und in der maraviglia eine Verbindung zum Metaphysischen und Göttlichen, wie sie schon von Fracastoro postuliert wurde. Patrizis Theorie des poetischen Furors ist ausführlicher ausgearbeitet als jene von Poliziano, Vida oder Fracastoro, sie gründet auf einer wissenschaftlichen Konzeption der anima und auf der platonischen Vorstellung des göttlichen Einflusses. Patrizis Furor-Theorie orientiert sich nicht an Platons Ion oder an Ficino und den damit verbundenen Vorstellungen der Übertragung, sondern wird über die psychologische Natur des Dichters begründet.

Allen bisher untersuchten Autoren ist die Begründung des Ursprungs der Dichtung im Mythos gemein, wobei Patrizi für die dichterischen Anfänge auf früheste, mythisch-fiktive Belege zurückgreift - wenngleich auch für ihn die Figur des Orpheus aufgrund der Ausrichtung seiner Poetik auf das Staunen zentral ist. Das Wunderbare - das mirabile - ist das Ziel jeder Dichtung, wobei der Dichter zu deren Gestaltung auf die Mittel der Transformation und der Fiktion und nicht auf die Nachahmung zurückgreift - Letztere dringt nicht zum « originario e privilegiato rapporto diretto con la radice prima del reale » vor. $^{155}$

Gänzlich neu ist die naturwissenschaftlich-philosophische Begründung der maraviglia in der potenza ammirativa. Sie lenkt die Aufmerksamkeit auf die Psychologie der Leser: Diese ist der Massstab aller Dinge, nur an ihr kann die dichterische Qualität orientiert und bemessen werden. Im Fokus von Patrizis Interesse steht in erster Linie der Mensch: als wahrnehmender und erkennender auf der einen Seite, als poetisch tätiger auf der anderen. Der Dichter definiert sich über die literarische Geschichte sowie seine ausserordentliche Konstitution - die Teilhabe am Göttlichen, Natürlichen und Künstlerischen. Diese erstaunlichen Charakteristika schlagen sich nieder im mirabile seiner Dichtung und in der maraviglia, die diese auslöst.

155 Bolzoni, L'universo, S. 29-30. 